DESY 01-154, IFUP-TH 2001/33

\title{
Critical exponents and equation of state of the three-dimensional Heisenberg universality class
}

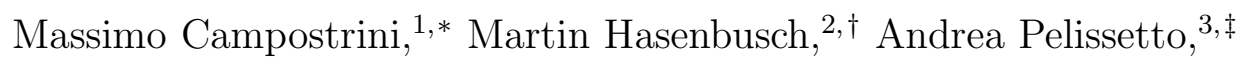 \\ Paolo Rossi, ${ }^{1}$ 具 and Ettore Vicari ${ }^{1,0}$ \\ ${ }^{1}$ Dipartimento di Fisica dell'Università di Pisa and I.N.F.N., I-56126 Pisa, Italy \\ ${ }^{2}$ NIC/DESY Zeuthen, Platanenallee 6, D-15738 Zeuthen, Germany \\ ${ }^{3}$ Dipartimento di Fisica dell'Università di Roma I and I.N.F.N., I-00185 Roma, Italy
}

\begin{abstract}
We improve the theoretical estimates of the critical exponents for the threedimensional Heisenberg universality class. We find $\gamma=1.3960(9), \nu=$ $0.7112(5), \eta=0.0375(5), \alpha=-0.1336(15), \beta=0.3689(3)$, and $\delta=4.783(3)$. We consider an improved lattice $\phi^{4}$ Hamiltonian with suppressed leading scaling corrections. Our results are obtained by combining Monte Carlo simulations based on finite-size scaling methods and high-temperature expansions. The critical exponents are computed from high-temperature expansions specialized to the $\phi^{4}$ improved model. By the same technique we determine the coefficients of the small-magnetization expansion of the equation of state. This expansion is extended analytically by means of approximate parametric representations, obtaining the equation of state in the whole critical region. We also determine a number of universal amplitude ratios.
\end{abstract}

PACS Numbers: 75.10.Hk, 75.10.-b, 05.70.Jk, 11.15.Me 
TABLE I. Recent experimental estimates of the critical exponents for Heisenberg systems.

\begin{tabular}{|c|c|c|c|c|}
\hline & Material & $\gamma$ & $\beta$ & $\delta$ \\
\hline 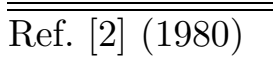 & $\mathrm{Ni}$ & & $0.354(14)$ & \\
\hline Ref. [3] (1981) & $\mathrm{Fe}$ & & $0.367(5)$ & \\
\hline Ref. 迎 (1995) & $\mathrm{Ni}$ & $1.345(10)$ & $0.395(10)$ & $4.35(6)$ \\
\hline Ref. [5] (1995) & $\mathrm{Gd}_{2} \mathrm{BrC}$ & $1.392(8)$ & $0.365(5)$ & $4.80(25)$ \\
\hline Ref. [5] (1995) & $\mathrm{Gd}_{2} \mathrm{IC}$ & $1.370(8)$ & $0.375(8)$ & $4.68(25)$ \\
\hline Ref. [6] (1999) & $\mathrm{Tl}_{2} \mathrm{Mn}_{2} \mathrm{O}_{7}$ & $1.31(5)$ & $0.44(6)$ & $4.65(15)$ \\
\hline Ref. [7] (2000) & $\mathrm{La}_{0.82} \mathrm{Ca}_{0.18} \mathrm{MnO}_{3}$ & & $0.383(9)$ & \\
\hline Ref. [8] (2000) & $\mathrm{La}_{0.95} \mathrm{Ca}_{0.05} \mathrm{MnO}_{3}$ & $1.39(5)$ & $0.36(7)$ & $4.75(15)$ \\
\hline Ref. [9] (2000) & $\operatorname{Gd}(0001)$ & & $0.376(15)$ & \\
\hline Ref. 110 (2000) & $\mathrm{Gd}_{2} \mathrm{CuO}_{4}$ & $1.32(2)$ & $0.34(1)$ & \\
\hline Ref. [1] (2000) & $\mathrm{C}_{80} \mathrm{Pd}_{20}$ (liq) & $1.42(5)$ & & \\
\hline Ref. [1] (2000) & $\mathrm{C}_{80} \mathrm{Pd}_{20}(\mathrm{sol})$ & $1.40(8)$ & & \\
\hline Ref. [12] (2001) & GdS & & $0.38(2)$ & \\
\hline Ref. 13] (2001) & $\mathrm{CrO}_{2}$ & $1.43(1)$ & $0.371(5)$ & \\
\hline Ref. 14 (2001) & $\mathrm{La}_{0.8} \mathrm{Ca}_{0.2} \mathrm{MnO}_{3}$ & 1.45 & 0.36 & \\
\hline
\end{tabular}

\section{INTRODUCTION AND SUMMARY}

According to the universality hypothesis, some features of continuous phase transitionsfor instance, critical exponents and scaling functions - do not depend on the microscopic details of the systems, but only on few global properties, such as the space dimensionality, the range of interaction, and the symmetry of the order parameter. These features define a universality class. In this paper, we consider the three-dimensional Heisenberg universality class, which is characterized by a three-component order parameter, $\mathrm{O}(3)$ symmetry, and short-range interactions.

The Heisenberg universality class describes [1] the critical behavior of isotropic magnets, for instance the Curie transition in isotropic ferromagnets such as $\mathrm{Ni}$ and $\mathrm{EuO}$, and of antiferromagnets such as $\mathrm{RbMnF}_{3}$ at the Néel transition point. In Table 国 we report some recent experimental results. It is not a complete review of published results, but it is useful to get an overview of the experimental state of the art. In the table we have also included results for the well-studied doped manganese perovskites $\mathrm{La}_{1-x} \mathrm{~A}_{x} \mathrm{MnO}_{3}$, although the nature of the ferromagnetic transition in these compounds is still unclear [15]. The Heisenberg universality class also describes isotropic magnets with quenched disorder. Indeed, since $\alpha<0$, the Harris criterion states that disorder is an irrelevant perturbation. The only effect is to introduce a correction-to-scaling term $|t|^{\Delta_{\text {dis }}}$ with $\Delta_{\text {dis }}=-\alpha$. The experimental results confirm the theoretical analysis [16], as it can be seen from Table II (older experimental results with a critical discussion are reported in Ref. [24]). The prediction for $\Delta_{\text {dis }}$ has been checked in perturbative field theory [25] and experimentally [26, 18, 19].

Beside the exponents $\gamma, \beta$, and $\delta$ there are also a few estimates of the specific-heat exponent $\alpha$, in most of the cases obtained from resistivity measurements: $\alpha \approx-0.10$ in Fe and $\mathrm{Ni}$ [27]; $\alpha=-0.12(2)$ in $\mathrm{EuO}$ [28]; $\alpha=-0.11(1)$ in $\mathrm{Fe}_{x} \mathrm{Ni}_{80-x} \mathrm{~B}_{19} \mathrm{Si}$ [17]; $\alpha=-0.11(1)$ 
TABLE II. Recent experimental estimates of the critical exponents for Heisenberg systems with quenched disorder.

\begin{tabular}{|c|c|c|c|c|}
\hline & Material & $\gamma$ & $\beta$ & $\delta$ \\
\hline Ref. 17] (1994) & $\mathrm{Fe}_{10} \mathrm{Ni}_{70} \mathrm{Bi}_{19} \mathrm{Si}$ & $1.387(12)$ & $0.378(15)$ & $4.50(5)$ \\
\hline Ref. [17 (1994) & $\mathrm{Fe}_{13} \mathrm{Ni}_{67} \mathrm{Bi}_{19} \mathrm{Si}$ & $1.386(12)$ & $0.367(15)$ & $4.50(5)$ \\
\hline Ref. 17] (1994) & $\mathrm{Fe}_{16} \mathrm{Ni}_{64} \mathrm{Bi}_{19} \mathrm{Si}$ & $1.386(14)$ & $0.360(15)$ & $4.86(4)$ \\
\hline Refs. 18,19 (1995) & $\mathrm{Fe}_{20} \mathrm{Ni}_{60} \mathrm{P}_{14} \mathrm{~B}_{6}$ & $1.386(10)$ & $0.367(10)$ & $4.77(5)$ \\
\hline Refs. [18, 19 (1995) & $\mathrm{Fe}_{40} \mathrm{Ni}_{40} \mathrm{P}_{14} \mathrm{~B}_{6}$ & $1.385(10)$ & $0.364(5)$ & $4.79(5)$ \\
\hline Ref. 20 (1997) & $\mathrm{Fe}_{91} \mathrm{Zr}_{9}$ & $1.383(4)$ & $0.366(4)$ & $4.75(5)$ \\
\hline Ref. 20] (1997) & $\mathrm{Fe}_{89} \mathrm{CoZr}_{10}$ & $1.385(5)$ & $0.368(6)$ & $4.80(4)$ \\
\hline Ref. 20 (1997) & $\mathrm{Fe}_{88} \mathrm{Co}_{2} \mathrm{Zr}_{10}$ & $1.389(6)$ & $0.363(5)$ & $4.81(5)$ \\
\hline Ref. 20] (1997) & $\mathrm{Fe}_{84} \mathrm{Co}_{6} \mathrm{Zr}_{10}$ & $1.386(6)$ & $0.370(5)$ & $4.84(5)$ \\
\hline Ref. [21] (1999) & $\mathrm{Fe}_{1.85} \mathrm{Mn}_{1.15} \mathrm{Si}$ & $1.543(20)$ & $0.408(60)$ & $4.74(7)$ \\
\hline Ref. [21] (1999) & $\mathrm{Fe}_{1.50} \mathrm{Mn}_{1.50} \mathrm{Si}$ & $1.274(60)$ & $0.383(10)$ & $4.45(19)$ \\
\hline Ref. [22] (2000) & $\mathrm{Fe}_{86} \mathrm{Mn}_{4} \mathrm{Zr}_{10}$ & 1.381 & 0.361 & \\
\hline Ref. [22] (2000) & $\mathrm{Fe}_{82} \mathrm{Mn}_{8} \mathrm{Zr}_{10}$ & 1.367 & 0.363 & \\
\hline Ref. 23. (2001) & $\mathrm{Fe}_{84} \mathrm{Mn}_{6} \mathrm{Zr}_{10}$ & $1.37(3)$ & 0.359 & $4.81(4)$ \\
\hline Ref. [23] (2001) & $\mathrm{Fe}_{74} \mathrm{Mn}_{16} \mathrm{Zr}_{10}$ & $1.39(5)$ & 0.361 & $4.86(3)$ \\
\hline
\end{tabular}

in $\mathrm{RbMnF}_{3}$ [29].

Aim of this paper is to substantially improve the precision of the theoretical estimates of the critical exponents. For this purpose, we consider an improved lattice Hamiltonian that is characterized by the fact that the leading correction to scaling is (approximately) absent in the expansion of any observable near the critical point. Moreover, we combine Monte Carlo (MC) simulations and analyses of high-temperature (HT) series. We exploit the effectiveness of $\mathrm{MC}$ simulations and finite-size scaling (FSS) techniques to determine the critical temperature and the parameters of the improved Hamiltonians [30 37], and the effectiveness of HT methods to determine the critical exponents for improved models, especially when a precise estimate of the critical point is available. This approach has already been applied to the three-dimensional Ising [38] and $X Y$ [39,36] universality classes, achieving a substantial improvement of the estimates of the universal quantities that describe the critical behavior, such as the critical exponents and the scaling equation of state.

We consider a simple cubic lattice and the nearest-neighbor $\phi^{4}$ lattice Hamiltonian

$$
\mathcal{H}_{\phi^{4}}=-\beta \sum_{\langle x y\rangle} \vec{\phi}_{x} \cdot \vec{\phi}_{y}+\sum_{x}\left[\vec{\phi}_{x}^{2}+\lambda\left(\vec{\phi}_{x}^{2}-1\right)^{2}\right],
$$

where $\vec{\phi}_{x}$ is a three-component field. As shown in Ref. [37], the Hamiltonian (11) is improved for $\lambda=\lambda^{*} \approx 4.4(7)$. Here, we extend the simulations of Ref. [37], obtaining a more accurate estimate of $\lambda^{*}, \lambda^{*}=4.6(4)$, and precise estimates of the critical $\beta_{c}$ for several values of $\lambda$. The analysis of the MC FSS results obtained for the improved $\phi^{4}$ lattice Hamiltonian already provides precise estimates of the critical exponents. As shown in Refs. [38, 39, 36, an additional increase in precision can be obtained by combining improved Hamiltonians and HT methods. For this purpose, by using the linked-cluster expansion technique, we 
computed HT expansions of several quantities and analyzed them using the MC results for $\lambda^{*}$ and $\beta_{c}$. The final results significantly improve those obtained from the MC simulation. Moreover, they substantially improve those obtained using longer (21 orders) series for the standard Heisenberg model [40].

In Table IIII we report our results for the critical exponents. We give the estimates obtained from the analysis of the $\mathrm{MC}$ data alone and those obtained by combining $\mathrm{MC}$ and HT techniques - they are denoted by MC+IHT, where the "I" refers to the fact that we are considering an improved model. The exponent $\alpha$ can be derived using the hyperscaling relation $\alpha=2-3 \nu$, obtaining $\alpha=-0.1336(15)$. We would like to stress that the good agreement between the MC and HT estimates is not trivial, since the critical exponents are determined from different quantities and limits. Indeed, the MC estimates are obtained from the analysis of the finite-size behavior for the size $L \rightarrow \infty$ at the critical point $\beta=\beta_{c}$, while the HT results are derived from the singular behavior of infinite-volume quantities as $\beta \rightarrow \beta_{c}$.

In Table III we compare our results with the most precise theoretical estimates that have been obtained in recent years. A more complete list of results can be found in Ref. [56]. The results we quote have been obtained by Monte Carlo simulations (MC), from the analysis of the HT series for the standard Heisenberg model (HT), or by field-theory methods (FT). The MC results were obtained by applying FSS techniques to different Hamiltonians. Refs. 42 [44 studied the standard $O(3)$-vector Heisenberg model, Ref. 37] the improved $\phi^{4}$ model, and Ref. [41] an isotropic ferromagnet with double-exchange interactions [57]. The HT results of Ref. [10] were obtained analyzing the 21st-order HT expansions for the standard $\mathrm{O}(3)$-vector model on the simple cubic (sc) and on the body-centered cubic (bcc) lattice. The FT results of Refs. [46 51] were derived by analyzing perturbative expansions in different frameworks: fixed-dimension expansion (6th- and 7th-order series, see Refs. [58,48]), $\epsilon$-expansion (to $O\left(\epsilon^{5}\right)$, see Refs. [59,60]), and $(d-2)$-expansion (to $O\left[(d-2)^{4}\right]$, see Refs. [61 63]). We quote two errors for the results of Ref. [18]: the first one (in parentheses) is the resummation error, and the second one (in brackets) takes into account the uncertainty of the fixed-point value $g^{*}$ of the coupling, which was estimated to be approximately $1 \%$ in Ref. [48]. To estimate the second error we use the results of Ref. [47] where the dependence of the exponents on $g^{*}$ is given. The results of Ref. [52 were obtained by using the so-called scaling-field method (SFM). Refs. [53 55.64] present results obtained by approximately solving continuous renormalization-group (CRG) equations for the average action, which is approximated to lowest and first order of the derivative expansion. We also mention the HT results of Ref. [65]: they performed a direct determination of the exponent $\alpha$ obtaining $\alpha=-0.11(2),-0.13(2)$ on the sc and bcc lattice. Ref. [66] computes the critical exponents for a Heisenberg fluid by a canonical-ensemble simulation. Depending on the analysis method, they find $1 / \nu=1.40(1), 1.31(1), \beta / \nu=0.54(2), 0.52(1)$, and $\gamma / \nu=1.90(3)$, 1.87(3). Overall, all estimates are in substantial agreement with our $\mathrm{MC}+\mathrm{IHT}$ results. We only note the apparent discrepancies with the MC estimates of $\eta$ of Refs. [42,43], and with the FT results of Ref. [46]. However, the reliability of the error bars reported in Ref. [46] is unclear: indeed, Ref. [47] analyzes the same perturbative series and reports much more cautious error estimates.

We also present a detailed study of the equation of state. We first consider its expansion in terms of the magnetization in the HT phase. The coefficients of this expansion are directly 
TABLE III. Estimates of the critical exponents. See text for the explanation of the symbols in the second column. We indicate with an asterisk $\left(^{*}\right)$ the estimates that have been obtained using the relations $\gamma=(2-\eta) \nu, 2 \beta=\nu(1+\eta), \delta(1+\eta)=5-\eta$.

\begin{tabular}{|c|c|c|c|c|c|c|}
\hline Ref. & Method & $\gamma$ & $\nu$ & $\eta$ & $\beta$ & $\delta$ \\
\hline this work & $\overline{\mathrm{MC}+\mathrm{IHT}}$ & 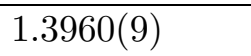 & $0.7112(5)$ & $0.0375(5)$ & 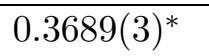 & $4.783(3)^{*}$ \\
\hline this work & $\mathrm{MC}$ & $1.3957(22)^{*}$ & $0.7113(11)$ & $0.0378(6)$ & $0.3691(6)^{*}$ & $4.781(3)^{*}$ \\
\hline [37] (2000) & $\mathrm{MC}$ & $1.393(4)^{*}$ & $0.710(2)$ & $0.0380(10)$ & $0.3685(11)^{*}$ & $4.780(6)^{*}$ \\
\hline [41] (2000) & $\mathrm{MC}$ & 1.3909(30) & $0.6949(38)$ & & $0.3535(30)$ & \\
\hline [42] (1996) & $\mathrm{MC}$ & $1.396(3)^{*}$ & $0.7128(14)$ & $0.0413(16)$ & $0.3711(9)^{*}$ & $4.762(9)^{*}$ \\
\hline (193) & $\mathrm{MC}$ & $1.389(14)^{*}$ & $0.704(6)$ & $0.027(2)$ & $0.362(3)^{*}$ & $4.842(11)^{*}$ \\
\hline [44] (1991) & $\mathrm{MC}$ & $1.390(23)^{*}$ & $0.706(9)$ & $0.031(7)$ & $0.364(5)^{*}$ & $4.82(4)^{*}$ \\
\hline [40] & $\mathrm{HT}$ sc & $1.406(3)$ & $0.716(2)$ & $0.036(7)^{*}$ & $0.3710(13)^{*}$ & $4.79(4)^{*}$ \\
\hline [40] (1997) & HT bcc & $1.402(3)$ & $0.714(2)$ & $0.036(7)^{*}$ & $0.3700(13)^{*}$ & $4.79(4)^{*}$ \\
\hline (1953) & HT & $1.40(1)$ & $0.712(10)$ & $0.03(3)^{*}$ & $0.368(6)^{*}$ & \\
\hline [4] $(2001)$ & $\mathrm{FT} d=3 \exp$ & $1.3882(10)$ & $0.7062(7)$ & $0.0350(8)$ & $0.3655(5)^{*}$ & $4.797(5)^{*}$ \\
\hline [47] (1998) & $\mathrm{FT} d=3 \exp$ & $1.3895(50)$ & $0.7073(35)$ & $0.0355(25)$ & $0.3662(25)$ & $4.794(14)$ \\
\hline [48] (1991) & $\mathrm{FT} d=3 \exp$ & $1.3926(13)[39]$ & $0.7096(8)[22]$ & $0.0374(4)$ & & \\
\hline [49] (1977) & $\mathrm{FT} d=3 \exp$ & $1.386(4)$ & $0.705(3)$ & $0.033(4)$ & $0.3645(25)$ & $4.808(22)$ \\
\hline [4] (1998) & FT $\epsilon$-exp & $1.382(9)$ & $0.7045(55)$ & $0.0375(45)$ & $0.3655(35)$ & $4.783(25)$ \\
\hline [50] (1998) & FT $\epsilon$-exp & $1.39^{*}$ & 0.708 & 0.037 & $0.367^{*}$ & $4.786^{*}$ \\
\hline [5] $(2000)$ & FT $(d-2)-\exp$ & & $0.695(10)$ & & & \\
\hline (52) & SFM & $1.40(3)$ & $0.715(20)$ & $0.044(7)$ & $0.373(11)$ & $4.75(4)^{*}$ \\
\hline [53] (2001) & CRG & & 0.74 & 0.038 & 0.37 & 4.78 \\
\hline [54] (2001) & CRG & 1.374 & 0.704 & 0.049 & 0.369 & 4.720 \\
\hline [55] (1996) & CRG & 1.465 & 0.747 & 0.038 & 0.388 & 4.78 \\
\hline
\end{tabular}

related to the zero-momentum $n$-point renormalized couplings, which are determined by analyzing their HT expansion. These results are used to construct parametric representations of the critical equation of state which are valid in the whole critical region, satisfy the correct analytic properties (Griffiths' analyticity), and take into account the Goldstone singularities at the coexistence curve. From our approximate representations of the equation of state we derive estimates of several universal amplitude ratios. Moreover, we present several results and different forms of the equation of state that can be compared directly with experiments. In particular, we can compare with the experimental results of Refs. [17, 20, 6], finding good agreement.

The paper is organized as follows. In Sec. [1] we present our MC results. In Sec. III we present our results for the critical exponents obtained from the analysis of the HT series for the improved Hamiltonian (11). The equation of state is discussed in Sec. IV. We determine the small-magnetization expansion coefficients in Sec. IV A, give an approximate parametric representation of the equation of state in Secs. [VB and IVC, compute several amplitude ratios in Sec. IVD, and compare the theoretical results with experimental data in Sec. IVE. Details are reported in the Appendices. In App. A we present the analysis of the MC results and in App. B the analysis of the HT series. The expressions of several amplitude ratios in 
TABLE IV. Final results for $\beta_{c}$ and $R^{*}$ from fits with ansatz (2). In parentheses we give the statistical error and in brackets the error due to the corrections to scaling.

\begin{tabular}{lllll}
\hline \hline$R$ & \multicolumn{1}{c}{$Z_{a} / Z_{p}$} & \multicolumn{1}{c}{$\xi_{2 \mathrm{nd}} / L$} & \multicolumn{1}{c}{$U_{4}$} & \multicolumn{1}{c}{$U_{6}$} \\
\hline \hline$R^{*}$ & $0.1944(1)[4]$ & $0.5644(1)[2]$ & $1.1394(1)[2]$ & $1.4202(2)[10]$ \\
$\beta_{c}$ & $0.6862390(10)[12]$ & $0.6862386(11)[6]$ & $0.6862365(17)[12]$ & $0.6862369(17)[19]$ \\
\hline \hline
\end{tabular}

terms of the parametric representations are reported in App. G.

\section{MONTE CARLO SIMULATIONS}

The present MC simulations extend those of Ref. [37]. Here, we have considerably enlarged the statistics and added larger lattice sizes. Moreover, we have considered an additional quantity in order to improve the control over systematic errors. This way, we can increase the accuracy of $\lambda^{*}$ and give precise estimates of the critical $\beta_{c}$ for three values of $\lambda$ in a neighborhood of $\lambda^{*}$. For a detailed discussion of our methods, see Ref. [36]. Details are reported in App. A.

We simulated the $O(3)$-symmetric $\phi^{4}$ model (1) at $\lambda=4.0,4.5$, and 5.0 on a simple cubic lattice with linear extension $L$ in all directions. We measured the Binder parameter $U_{4}$, its sixth-order generalization $U_{6}$, the second-moment correlation length $\xi_{2 \text { nd }}$, and the ratio $Z_{a} / Z_{p}$, where $Z_{a}$ is the partition function with anti-periodic boundary conditions in one of the three directions and $Z_{p}$ the corresponding one with periodic boundary conditions in all directions. The number of iterations for each lattice size and value of $\lambda$ was approximately $10^{7}$ for $L=6,7,8,9,10,11,12,14,16,18,20,22$, approximately $10^{6}$ for $L=24,28,32,36,40,48$, and $1-4 \times 10^{5}$ for $L=56,64,80,96$. With respect to Ref. [37], we have added new lattice sizes for all three values of $\lambda$ and considerably increased the statistics. In total, the whole study took about four years on a single $450 \mathrm{MHz}$ Pentium III CPU.

In the first step of the analysis, we compute $\beta_{c}$ and the fixed-point value of the dimensionless ratios $R^{*}$ for $\lambda=4.5$, using the standard cumulant crossing method of Binder. In particular, we fit our data with the ansatz

$$
R^{*}=R\left(L, \beta_{c}\right),
$$

where $R^{*}$ and $\beta_{c}$ are free parameters. Our results are reported in Table $\mathbb{\mathbb { V }}$. Note that the four results for $\beta_{c}$ are consistent within error bars. The statistical error of $\beta_{c}$ obtained from $Z_{a} / Z_{p}$ and $\xi_{2 \text { nd }} / L$ is considerably smaller than that from $U_{4}$ and $U_{6}$. As our final estimate we take $\beta_{c}=0.6862385(20)$, which is consistent with all four results.

In addition, we determine $\beta_{c}$ for $\lambda=4.0$ and $\lambda=5.0$. For this purpose, we use the ansatz (2), fixing $L=96$ and taking the values of $R^{*}$ from Table $\mathbb{I V}$. Our results are summarized in Table $\nabla$. For both values of $\lambda$, the results obtained from the four different choices of $R^{*}$ are consistent within error bars. As our final result we take that obtained from $Z_{a} / Z_{p}$, since it has the smallest statistical error.

Then, we locate $\lambda^{*}$ by studying the scaling corrections to a quantity $\bar{R}$ defined in terms of two dimensionless ratios $R_{1}$ and $R_{2}$. To define $\bar{R}$, we fix a number $R_{1, f}$ which should be a 
TABLE V. Results for $\beta_{c}$ at $\lambda=4.0$ and 5.0 using only $L=96$ and the ansatz $R\left(\beta_{c}\right)=R^{*}$, where $R^{*}$ is taken from Table IV. In parentheses we give the statistical error and in brackets the error due to the uncertainty on $R^{*}$.

\begin{tabular}{ccccc}
\hline \hline$\lambda$ & $Z_{a} / Z_{p}$ & $\xi_{2 \text { nd }} / L$ & $U_{4}$ & $U_{6}$ \\
\hline \hline 4.0 & $0.6843895(20)[15]$ & $0.6843887(21)[14]$ & $0.6843898(31)[20]$ & $0.6843898(31)[26]$ \\
5.0 & $0.6875638(21)[16]$ & $0.6875633(26)[15]$ & $0.6875655(34)[20]$ & $0.6875646(34)[26]$ \\
\hline \hline
\end{tabular}

good approximation to $R_{1}^{*}$, see Ref. [36]. Then, for a given value of $\lambda$ and $L$, we determine $\beta_{f}(L, \lambda)$ from

$$
R_{1}\left(L, \lambda, \beta_{f}\right)=R_{1, f} .
$$

In our analysis, $\beta_{f}$ is determined by taking either $\left(Z_{a} / Z_{p}\right)_{f}=0.1944$ or $\left(\xi_{2 \text { nd }} / L\right)_{f}=0.5644$. Note that $\beta_{f}$ approaches $\beta_{c}$ as

$$
\beta_{f}=\beta_{c}+C_{f} L^{-1 / \nu}+\ldots
$$

where the prefactor $C_{f}$ depends on the choice of $R_{1, f}$. In particular, if $R_{1, f}=R_{1}^{*}$, then $C_{f}=0$ and the leading corrections are proportional to $L^{-1 / \nu-\omega}$.

Next, we define $\bar{R}$ by

$$
\bar{R}(L, \lambda) \equiv R_{2}\left(L, \lambda, \beta_{f}\right) .
$$

Here, we take either $U_{4}$ or $U_{6}$ as $R_{2}$. Below, we often refer to $\bar{R}$ as $R_{2}$ at $R_{1, f}$. Up to subleading corrections, $\bar{R}$ behaves as

$$
\bar{R}(L, \lambda) \approx \bar{R}^{*}+\bar{c}(\lambda) L^{-\omega}
$$

The optimal value $\lambda^{*}$ is obtained by solving $\bar{c}(\lambda)=0$. We obtain $\lambda^{*}=4.6(4), 4.7(8), 4.7(8)$ and 4.6(8) from $U_{4}$ at $\left(Z_{a} / Z_{p}\right)_{f}=0.1944, U_{4}$ at $\left(\xi_{\text {2nd }} / L\right)_{f}=0.5644, U_{6}$ at $\left(Z_{a} / Z_{p}\right)_{f}=0.1944$ and $U_{6}$ at $\left(\xi_{2 n d} / L\right)_{f}=0.5644$, respectively. As our final result we quote

$$
\lambda^{*}=4.6(4)
$$

from $U_{4}$ at $\left(Z_{a} / Z_{p}\right)_{f}=0.1944$.

Finally, we compute the critical exponents $\nu$ and $\eta$ using standard FSS methods. Usually, the exponent $\nu$ is computed from the slope of a dimensionless ratio $R$ at $\beta_{c}$. Here, following Ref. [42], we replace $\beta_{c}$ by $\beta_{f}$, which simplifies the error analysis, and determine $\nu$ from the relation

$$
\left.\frac{\partial R}{\partial \beta}\right|_{\beta_{f}}=\bar{a} L^{1 / \nu} .
$$

We study the derivative of all four quantities $U_{4}, U_{6}, \xi_{2 \text { nd }} / L$, and $Z_{a} / Z_{p}$, and fix $\beta_{f}$ by using either $\left(\xi_{\text {2nd }} / L\right)_{f}=0.5644$ or $\left(Z_{a} / Z_{p}\right)_{f}=0.1944$. We arrive at the final estimate

$$
\nu=0.7113(11),
$$


where the error includes both the statistical and the systematic uncertainty.

The exponent $\eta$ is computed from the finite-size behavior of the magnetic susceptibility:

$$
\left.\chi\right|_{\beta_{f}}=c L^{2-\eta} .
$$

In addition, we also use a fit ansatz that includes a constant background term:

$$
\left.\chi\right|_{\beta_{f}}=c L^{2-\eta}+b .
$$

As before, we fix $\beta_{f}$ by setting either $\left(\xi_{2 \text { nd }} / L\right)_{f}=0.5644$ or $\left(Z_{a} / Z_{p}\right)_{f}=0.1944$. Our final $\mathrm{MC}$ estimate is

$$
\eta=0.0378(6) .
$$

\section{CRITICAL EXPONENTS FROM THE IMPROVED HIGH-TEMPERATURE EXPANSION}

As shown in the case of the Ising [38] and $X Y$ universality classes [39, 36], the analysis of HT expansions for improved Hamiltonians with suppressed leading scaling corrections leads to considerably precise results even for moderately long series. In the present paper, the analysis of 20th-order HT expansions for the improved $\phi^{4}$ lattice Hamiltonian, i.e. for $\lambda \approx \lambda^{*}=4.6(4)$, allows us to substantially improve the accuracy of the estimates of the critical exponents. As we shall see, the results turn out to be more precise than those obtained in the preceding Section. They also significantly improve those obtained from the analysis of longer series (21 orders) for the standard Heisenberg model (which is recovered in the limit $\lambda \rightarrow \infty$ ) on the cubic and bcc lattices [10]. In this Section we report the results of our analyses of the HT series. The details are reported in App. B.

We determine $\gamma$ and $\nu$ from the analysis of the HT expansion to $O\left(\beta^{20}\right)$ of the magnetic susceptibility and of the second-moment correlation length. In App. B2 we report some details and intermediate results so that the reader can judge the quality of our results without the need of performing his own analysis. They should give an idea of the reliability of our estimates and of the meaning of the errors we quote, which depend on many somewhat arbitrary choices and are therefore partially subjective.

We analyze the HT series by means of integral approximants (IA's) of first, second, and third order. The most precise results are obtained biasing the value of $\beta_{c}$ with its MC estimate. We consider several sets of biased IA's and for each of them we obtain estimates of the critical exponents. These results are reported in App. B2. All sets of IA's give substantially consistent results. Moreover, the results are also stable with respect to the number of terms of the series, so that there is no need to perform problematic extrapolations in the number of terms in order to obtain the final estimates. The error due to the uncertainty on $\lambda^{*}$ is estimated by considering the variation of the results when changing the values of $\lambda$.

Using the results reported in App. B2 for the analysis at $\lambda=4.0,4.5$, and 5.0, we obtain

$$
\begin{aligned}
& \gamma=1.39582(10)[18]+0.0015(\lambda-4.5), \\
& \nu=0.71111(5)[8]+0.0009(\lambda-4.5) .
\end{aligned}
$$


The number between parentheses is basically the spread of the approximants at $\lambda=4.5$ using the central value of $\beta_{c}$, while the number between brackets gives the systematic error due to the uncertainty on $\beta_{c}$. Eqs. (13) and (14) show also the dependence of the results on the chosen value of $\lambda$. The $\lambda$-dependence is estimated by using the results for $\lambda=4.0$ and $\lambda=5.0$.

Using the $\mathrm{MC}$ estimate $\lambda^{*}=4.6(4)$, we obtain

$$
\begin{aligned}
& \gamma=1.39597(10)[18]\{60\} \\
& \nu=0.71120(5)[8]\{36\},
\end{aligned}
$$

where the error due to the uncertainty on $\lambda^{*}$ is reported between braces. Thus, our final estimates are

$$
\begin{aligned}
& \gamma=1.3960(9) \\
& \nu=0.7112(5)
\end{aligned}
$$

where the uncertainty is estimated by summing the three errors reported above.

Using the above-reported results for $\gamma$ and $\nu$ and the scaling relation $\gamma=(2-\eta) \nu$, we obtain $\eta=0.037(2)$, where the error is estimated by considering the errors on $\gamma$ and $\nu$ as independent, which is of course not true. We can obtain an estimate of $\eta$ with a smaller, yet reliable, error by applying the so-called critical-point renormalization method 67 to the series of $\chi$ and $\xi^{2}$. This method provides an estimate for the combination $\eta \nu$. Proceeding as before, we obtain

$$
\eta \nu=0.02665(18)+0.00035(\lambda-4.5) \text {. }
$$

Taking into account that $\lambda^{*}=4.6(4)$, we find

$$
\eta \nu=0.02669(18)[14]
$$

where the first error is related to the spread of the IA's and the second one to the uncertainty on $\lambda^{*}$, evaluated as before. Thus,

$$
\eta=0.0375(3)[2]
$$

Moreover, using the scaling relations, one obtains

$$
\begin{aligned}
\alpha & =2-3 \nu=-0.1336(15), \\
\delta & =\frac{5-\eta}{1+\eta}=4.783(3), \\
\beta & =\frac{\nu}{2}(1+\eta)=0.3689(3),
\end{aligned}
$$

where the error of $\beta$ has been estimated by considering the errors of $\nu$ and $\eta$ as independent. 


\section{THE CRITICAL EQUATION OF STATE}

In this section we determine the critical equation of state characterizing the Heisenberg universality class. The critical equation of state relates the thermodynamical quantities in the neighborhood of the critical temperature, in both phases. It is usually written in the form

$$
\begin{aligned}
& \vec{H}=\left(B_{c}\right)^{-\delta} \vec{M} M^{\delta-1} f(x), \\
& x \equiv t(M / B)^{-1 / \beta}
\end{aligned}
$$

where $f(x)$ is a universal scaling function normalized in such a way that $f(-1)=0$ and $f(0)=1$, and $B_{c}$ and $B$ are the amplitudes of the magnetization on the critical isotherm and on the coexistence curve,

$$
\begin{array}{ll}
M=B_{c} H^{1 / \delta} & t=0, \\
M=B(-t)^{\beta} & H=0, t<0 .
\end{array}
$$

Griffiths' analyticity implies that $f(x)$ is regular everywhere for $x>-1$. It has a regular expansion in powers of $x$,

$$
f(x)=1+\sum_{n=1}^{\infty} f_{n}^{0} x^{n}
$$

and a large- $x$ expansion of the form

$$
f(x)=x^{\gamma} \sum_{n=0}^{\infty} f_{n}^{\infty} x^{-2 n \beta} .
$$

Moreover, at the coexistence curve, i.e. for $x \rightarrow-168$

$$
f(x) \approx c_{f}(1+x)^{2} .
$$

The nature of the corrections to the leading behavior at the coexistence curve is less clear, see, e.g., Refs. [70 73,56]. From the scaling function $f(x)$ one may derive many interesting universal amplitude ratios involving zero-momentum quantities, such as specific heat, magnetic susceptibility, etc.... For example, the universal ratio $U_{0}$ of the specific-heat amplitudes in the two phases can be written as (see, e.g., Ref. [74])

$$
U_{0} \equiv \frac{A^{+}}{A^{-}}=\frac{\varphi(\infty)}{\varphi(-1)}
$$

where, in the Heisenberg case for which $-1<\alpha<0$,

$$
\varphi(x)=\frac{x|x|^{\alpha-2} f^{\prime}(0)}{\alpha-1}+\frac{|x|^{\alpha} f^{\prime \prime}(0)}{\alpha}-|x|^{\alpha-2} f(x)+\int_{0}^{x} d y|y|^{\alpha-2}\left[f^{\prime}(y)-f^{\prime}(0)-y f^{\prime \prime}(0)\right] \text {. }
$$

We mention that the critical equation of state for the $N$-vector model has been computed to $O\left(\epsilon^{2}\right)$ in the framework of the $\epsilon$-expansion [75] and to $O(1 / N)$ in the framework of the $1 / N$ expansion 68]. 
As our starting point for the determination of the critical equation of state, we determine the first few nontrivial coefficients of its small-magnetization expansion, by analyzing the corresponding HT series for the improved $\phi^{4}$ Hamiltonian. These results are then used to construct parametric representations of the critical equation of state which are valid in the whole critical region. Then, from our approximate representations of the equation of state we derive estimates of several universal amplitude ratios. This method has been already applied to the Ising universality class in three [76, 38] and two dimensions [77], and to the three-dimensional $X Y$ universality class [78,36.

\section{A. Small-magnetization expansion of the Helmholtz free energy}

We write the Helmholtz free energy as [76, 79

$$
\Delta \mathcal{F}=\mathcal{F}(M)-\mathcal{F}_{\text {reg }}(M)=\frac{m^{3}}{g_{4}} A(z)
$$

where $m=1 / \xi$, $\xi$ is the second-moment correlation length, $g_{4}$ is the zero-momentum fourpoint coupling, and

$$
z \equiv k|M| t^{-\beta}
$$

where $k$ is an appropriate amplitude ratio. The small-magnetization expansion of the free energy corresponds to the small- $z$ expansion of $A(z)$,

$$
A(z)=\frac{1}{2} z^{2}+\frac{1}{4 !} z^{4}+\sum_{j=3} \frac{1}{(2 j) !} r_{2 j} z^{2 j}
$$

which also fixes the normalization $k$ of $z$. Correspondingly, we obtain for the equation of state

$$
\vec{H}=\frac{\partial \mathcal{F}(M)}{\partial \vec{M}} \propto \frac{\vec{M}}{|M|} t^{\beta \delta} F(z),
$$

with $F(z) \equiv \partial A / \partial z$. Because of Griffiths' analyticity, $\mathcal{F}(M)$ has a regular expansion in powers of $t$ for $|M|$ fixed. Therefore, $F(z)$ has the large- $z$ expansion

$$
F(z)=z^{\delta} \sum_{k=0} F_{k}^{\infty} z^{-k / \beta}
$$

The function $F(z)$ is defined only for $t>0$. For $t<0$ the equation of state is expressed in terms of a different function which is however analytically related to $F(z)$ since the free energy and the equation of state are analytic on the critical isotherm $t=0$ for $H \neq 0$. The two functions $f(x)$ and $F(z)$ are clearly related:

$$
z^{-\delta} F(z)=F_{0}^{\infty} f(x), \quad z=z_{0} x^{-\beta}
$$

where $z_{0}=k B$. 
TABLE VI. Estimates of $g_{4}, r_{6}$, and $r_{8}$, obtained using various approaches.

\begin{tabular}{|c|c|c|c|c|c|}
\hline & IHT & $\mathrm{HT}$ & $d=3$ exp. & $\epsilon$-exp. & $\mathrm{CRG}$ \\
\hline$g_{4}$ & $19.13(10)$ & $\begin{array}{l}19.31(14), 19.27(11) \\
19.34(16)\end{array}$ & $\begin{array}{l}19.06(5)[47] \\
19.06[48\end{array}$ & $19.55(12)$ 81,82 & 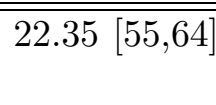 \\
\hline$r_{6}$ & $1.86(4)$ & $2.1(6)$ 83] & $\begin{array}{l}1.880 \text { 84 } \\
1.884(32)\end{array}$ & $1.867(9) 81,85$ & 1.7486 \\
\hline$r_{8}$ & $0.6(2)$ & & $0.975[84]$ & $1.0(6)$ [81, 85, & 0.84 86 \\
\hline
\end{tabular}

In order to estimate the universal quantities $g_{4}$ and $r_{2 j}$ from the corresponding improved HT expansions (see App. B 1), we essentially used the analysis described in Ref. [36]. Here, we report only the final estimates:

$$
\begin{aligned}
& g_{4}=19.13(8)[2], \\
& r_{6}=1.86(3)[1], \\
& r_{8}=0.60(15)[5],
\end{aligned}
$$

where the error in parentheses is related to the spread of the approximants and the second one in brackets to the uncertainty on $\lambda^{*}$, evaluated as before. Moreover, we obtained the rough estimate $r_{10}=-15(10)$. In Table VI we compare our results (denoted by IHT) with the estimates obtained using other approaches, such as HT expansions for the standard $O(3)$-vector model $(\mathrm{HT})$, field-theoretical fixed-dimension perturbative expansions $(d=3$

exp.), $\epsilon$ expansions ( $\epsilon$-exp.), and approximate solutions of continuous renormalization-group equations (CRG). All estimates are in good agreement, Only the $\epsilon$-expansion estimate of $g_{4}$ is significantly higher than the IHT estimate (as already noted in Ref. [81], the error may be underestimated). The CRG estimates are much less precise than the results of other methods.

\section{B. Parametric representations of the equation of state}

In order to obtain approximations of the equation of state valid in the whole critical region, we use parametric representations that implement the expected scaling and analytic properties. We write 8789

$$
\begin{aligned}
M & =m_{0} R^{\beta} m(\theta), \\
t & =R\left(1-\theta^{2}\right), \\
H & =h_{0} R^{\beta \delta} h(\theta),
\end{aligned}
$$

where $h_{0}$ and $m_{0}$ are normalization constants. The variable $R$ is nonnegative and measures the distance from the critical point in the $(t, H)$ plane, while the variable $\theta$ parametrizes the displacement along the lines of constant $R$. The functions $m(\theta)$ and $h(\theta)$ are odd and normalized so that $m(\theta)=\theta+O\left(\theta^{3}\right)$ and $h(\theta)=\theta+O\left(\theta^{3}\right)$. The smallest positive zero of $h(\theta)$, which should satisfy $\theta_{0}>1$, corresponds to the coexistence curve, i.e., to $T<T_{c}$ and $H \rightarrow 0$. The parametric representation satisfies the requirements of regularity of the 
equation of state. Singularities can appear only at the coexistence curve (due, for example, to the logarithms discussed in Ref. [73]), i.e., for $\theta=\theta_{0}$. The mapping (43]) is not invertible when its Jacobian vanishes, which occurs when

$$
Y(\theta) \equiv\left(1-\theta^{2}\right) m^{\prime}(\theta)+2 \beta \theta m(\theta)=0
$$

Thus, parametric representations based on the mapping (43) are acceptable only if $\theta_{0}<\theta_{l}$ where $\theta_{l}$ is the smallest positive zero of the function $Y(\theta)$.

The functions $m(\theta)$ and $h(\theta)$ are related to the scaling function $f(x)$ through

$$
\begin{aligned}
& x=\frac{1-\theta^{2}}{\theta_{0}^{2}-1}\left[\frac{m\left(\theta_{0}\right)}{m(\theta)}\right]^{1 / \beta}, \\
& f(x)=\left[\frac{m(\theta)}{m(1)}\right]^{-\delta} \frac{h(\theta)}{h(1)} .
\end{aligned}
$$

The asymptotic behavior (31) is reproduced simply by requiring that

$$
h(\theta) \sim\left(\theta_{0}-\theta\right)^{2} \quad \text { for } \quad \theta \rightarrow \theta_{0} .
$$

The scaling function $F(z)$ is obtained by

$$
\begin{aligned}
& z=\rho m(\theta)\left(1-\theta^{2}\right)^{-\beta}, \\
& F(z(\theta))=\rho\left(1-\theta^{2}\right)^{-\beta \delta} h(\theta),
\end{aligned}
$$

where $\rho$ may be taken as a free parameter [76, 38, 78, 56$]$.

\section{Approximate polynomial representations}

Following Ref. [78, we construct approximate polynomial parametric representations that have the expected singular behavior at the coexistence curve (Goldstone singularity) and match the known terms of the small- $z$ expansion of $F(z)$, cf. Eqs. (36) and (37). We

consider two distinct approximation schemes. In the first one, which we denote by $(\mathrm{A}), h(\theta)$ is a polynomial of fifth order with a double zero at $\theta_{0}$, and $m(\theta)$ is a polynomial of order $(1+2 n)$ :

$$
\begin{aligned}
& \text { scheme }(\mathrm{A}): \quad m(\theta)=\theta\left(1+\sum_{i=1}^{n} c_{i} \theta^{2 i}\right) \text {, } \\
& h(\theta)=\theta\left(1-\theta^{2} / \theta_{0}^{2}\right)^{2} \text {. }
\end{aligned}
$$

In the second scheme, denoted by $(\mathrm{B})$, we set

$$
\begin{aligned}
m(\theta) & =\theta \\
\text { scheme (B): } \quad & \\
h(\theta) & =\theta\left(1-\theta^{2} / \theta_{0}^{2}\right)^{2}\left(1+\sum_{i=1}^{n} c_{i} \theta^{2 i}\right) .
\end{aligned}
$$


Here $h(\theta)$ is a polynomial of order $5+2 n$ with a double zero at $\theta_{0}$. For $n=0$ the approximations (A) and (B) coincide. Note that for scheme (B)

$$
Y(\theta)=1-\theta^{2}+2 \beta \theta^{2},
$$

independently of $n$, so that $\theta_{l}=(1-2 \beta)^{-1}$. In both schemes, $\rho, \theta_{0}$, and the $n$ coefficients $c_{i}$ are determined by matching the small- $z$ expansion of $F(z)$. Thus, in order to fix the $n$ coefficients $c_{i}$ we use $n+1$ values of $r_{2 j}$, i.e., $r_{6}, \ldots r_{6+2 n}$.

As input parameters for our analysis we consider the estimates $\alpha=-0.1336(15), \eta=$ $0.0375(5), r_{6}=1.86(4)$, and $r_{8}=0.6(2)$, which are the results of our HT analysis. The available estimate of $r_{10}$ is too imprecise for our purposes. In Fig. 1 we show the curves obtained in schemes (A) and (B) with $n=0,1$ and for $\alpha=-0.1336, \eta=0.0375, r_{6}=1.86$, and $r_{8}=0.6$. The differences among the three approximations should give an indication of the uncertainty. The three approximations of $F(z)$ are practically indistinguishable, and differ at most by approximately $2 \%$ (the difference between the two $n=1$ curves is much smaller). Thus, by using the first two coefficients $r_{2 j}$, one obtains reasonably precise approximations of the scaling function $F(z)$ for all positive values of $z$, i.e., for the whole HT phase up to $t=0$. This is also numerically confirmed by the estimates of the universal constant $F_{0}^{\infty}$, cf. Eq. (38), which is related to the large- $z$ behavior of $F(z)$. Indeed, we obtain $F_{0}^{\infty}=0.0262(4), 0.0266(5), 0.0266(5)$ respectively for $n=0, n=1(\mathrm{~A}), n=1$ (B), where the reported errors refer only to the uncertainty of the input parameters. This fact is not trivial, since the small- $z$ expansion has a finite convergence radius [90]. Therefore, the determination of $F(z)$ on the whole positive real axis from its small- $z$ expansion requires an analytic continuation, which turns out to be effectively performed by the approximate parametric representations we have considered.

In Fig. 2 we plot the approximations of $f(x)$ corresponding to the schemes $(\mathrm{A})$ and (B) for $n=0,1$, using the central values of the input parameters. The three curves are in substantial agreement, especially those with $n=1$. Indeed, the difference between them is within the uncertainty due to the errors on the input parameters. These approximate parametric representations are not precise at the coexistence curve; indeed, as we shall see, the estimates of $c_{f}$, cf. Eq. (31), are rather imprecise and very sensitive to the value of $r_{8}$, which is not known with high precision. We mention that in Ref. [55] an approximate expression for $f(x)$ was obtained by approximately solving the continuous renormalizationgroup equations for the free energy (average action). The results are quite imprecise, as we shall show later by comparing the corresponding estimates for some universal amplitude ratios.

\section{Universal amplitude ratios}

From the critical equation of state one may derive estimates of several universal amplitude ratios. They are expressed in terms of the amplitudes of the magnetization, cf. Eqs. (27) and (28), of the singular part of the specific heat

$$
C_{H, \text { sing }}=A^{ \pm}|t|^{-\alpha}
$$

of the magnetic susceptibility in the HT phase 


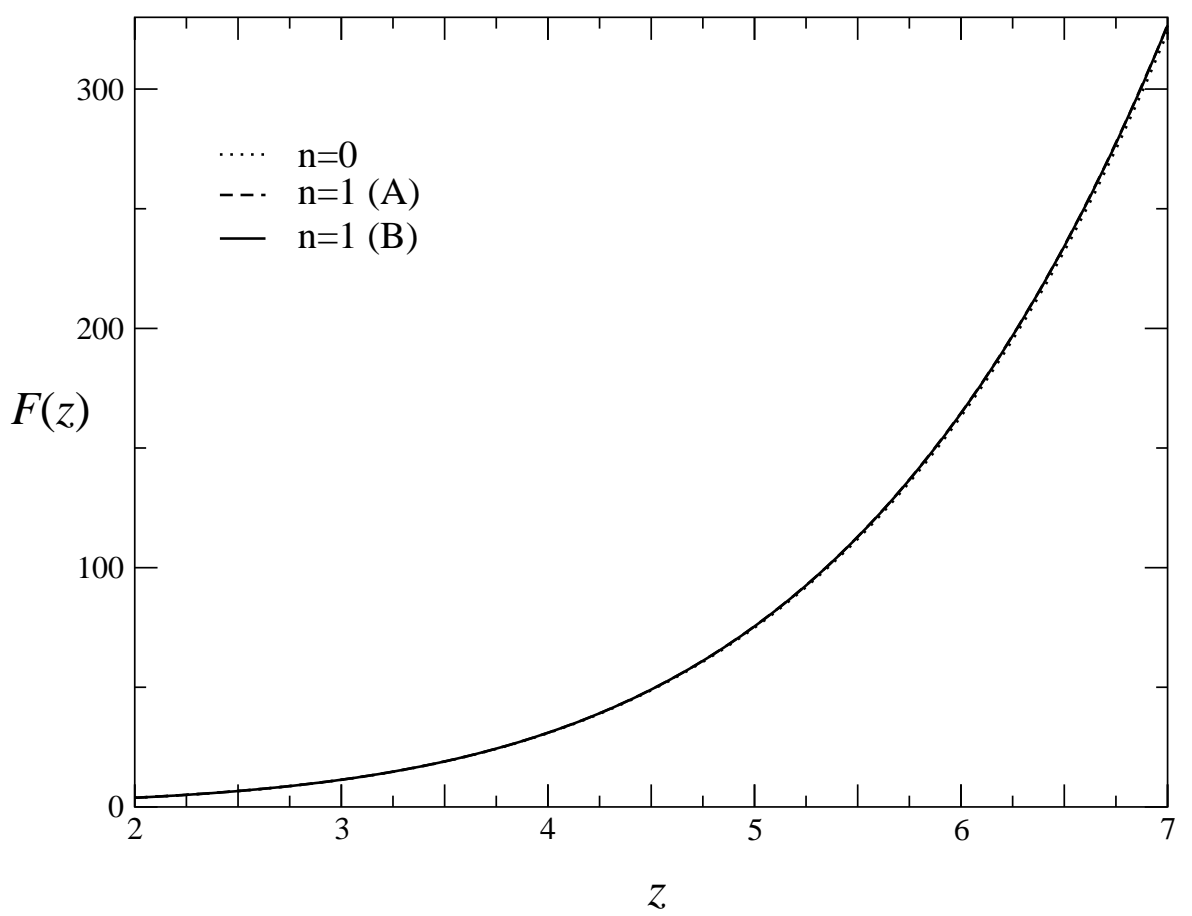

FIG. 1. The scaling function $F(z)$ versus $z$.

$$
\chi=N C^{+} t^{-\gamma}
$$

of the zero-momentum four-point connected correlation function in the HT phase

$$
\chi_{4}=\frac{N(N+2)}{3} C_{4}^{+} t^{-\gamma-2 \beta \delta}
$$

and of the second-moment correlation length in the HT phase

$$
\xi=f^{+} t^{-\nu}
$$

where $N=3$. We also consider the crossover (or pseudocritical) line $t_{\text {max }}(H)$, that is defined as the reduced temperature for which the longitudinal magnetic susceptibility $\chi_{L}(t, H)=$ $\partial M / \partial H$ has a maximum at $H$ fixed. The renormalization group predicts

$$
\begin{aligned}
& t_{\max }(H)=T_{p} H^{1 /(\gamma+\beta)}, \\
& \chi_{L}\left(t_{\max }, H\right)=C_{p} t_{\max }^{-\gamma} .
\end{aligned}
$$

We consider several universal amplitude ratios:

$$
\begin{aligned}
& U_{0} \equiv \frac{A^{+}}{A^{-}}, \\
& R_{\chi} \equiv \frac{C^{+} B^{\delta-1}}{\left(B_{c}\right)^{\delta}}, \\
& R_{C} \equiv \frac{\alpha A^{+} C^{+}}{B^{2}},
\end{aligned}
$$




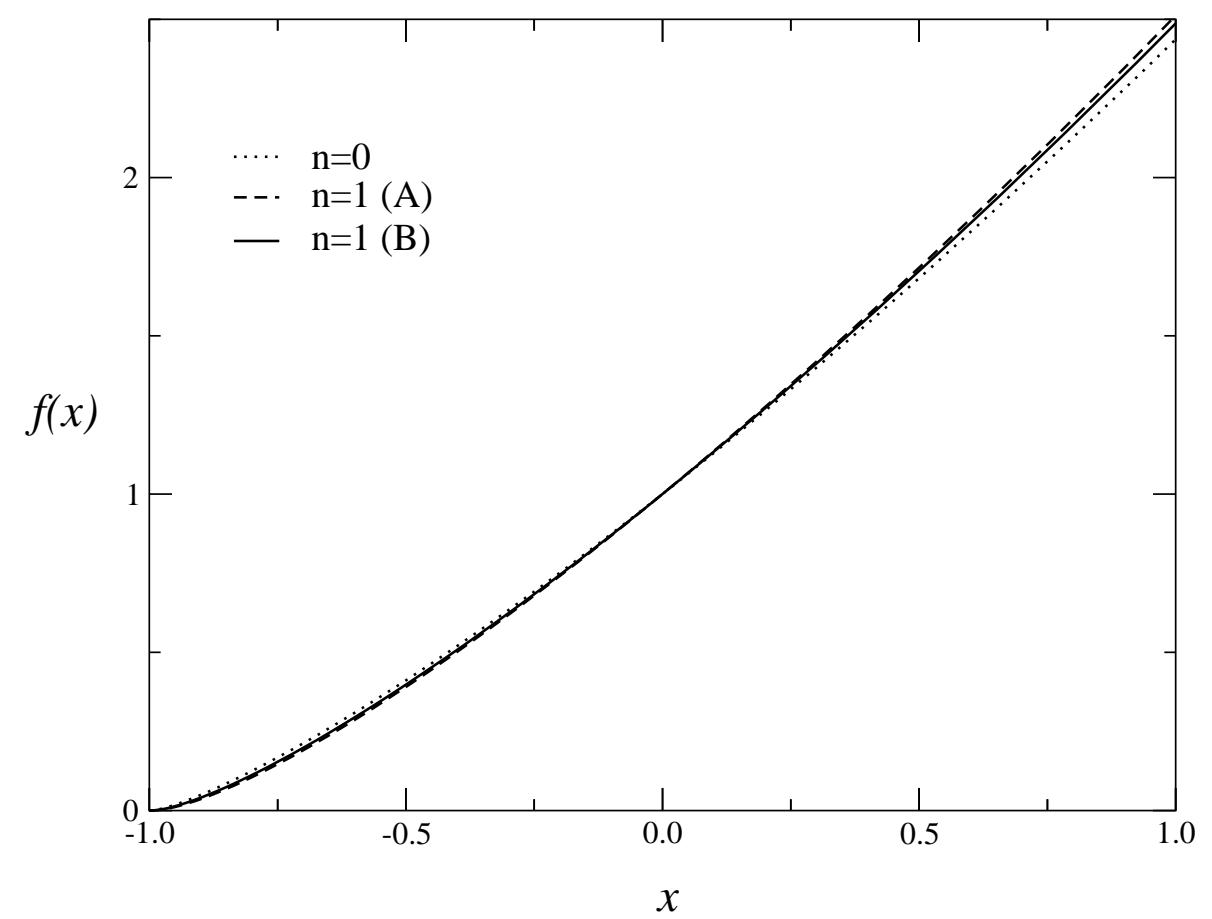

FIG. 2. The scaling function $f(x)$ versus $x$.

$$
\begin{aligned}
R_{4} & \equiv-\frac{C_{4}^{+} B^{2}}{\left(C^{+}\right)^{3}} \\
R_{\xi}^{+} & \equiv\left(\alpha A^{+}\right)^{1 / 3} f^{+}=\left(\frac{R_{C} R_{4}}{g_{4}}\right)^{1 / 3}, \\
P_{m} & \equiv \frac{T_{p}^{\beta} B}{B_{c}} \\
P_{c} & \equiv-\frac{T_{p}^{2 \beta \delta} C^{+}}{C_{4}^{+}}=\frac{P_{m}^{2 \delta}}{R_{\chi}^{2} R_{4}}, \\
R_{p} & \equiv \frac{C^{+}}{C_{p}}
\end{aligned}
$$

Morever, we estimate

$$
R_{\alpha} \equiv \frac{1-U_{0}}{\alpha},
$$

which, as suggested in Ref. [74], should be less sensitive to the value of $\alpha$ than $U_{0}$. In App. 9 we give their expressions in terms of the functions $m(\theta)$ and $h(\theta)$.

In Table VII we report the universal amplitude ratios, as derived by the approximate polynomial representations of the equation of state for $n=0,1$. The reported errors are only due to the uncertainty of the input parameters and do not include the systematic error of the procedure, which may be determined by comparing the results of the various approximations. In Table VII we also show results for $z_{\max }, x_{\max }$ and $w_{\max }$ which are the values of the scaling variable $z, x$ and $w$ ( $w$ will be defined in Eq. (71)) associated with the 
TABLE VII. Results for the parameters and the universal amplitude ratios using the scheme (A), cf. Eq. (48), and the scheme (B), cf. Eq. (49). Note that the quantities reported in the first three lines do not have a physical meaning, but are related to the particular parametric representation employed. Numbers marked with an asterisk are inputs, not predictions.

\begin{tabular}{lccc}
\hline \hline & $n=0$ & $n=1(\mathrm{~A})$ & $n=1(\mathrm{~B})$ \\
\hline \hline$\rho$ & $2.14(2)$ & $2.20(4)$ & $2.08(4)$ \\
$\theta_{0}^{2}$ & $3.81(1)$ & $3.3(1)$ & $2.7(2)$ \\
$c_{1}$ & 0 & $-0.016(9)$ & $0.055(20)$ \\
\hline$U_{0}$ & $1.61(2)$ & $1.56(3)$ & $1.57(3)$ \\
$R_{\alpha}$ & $4.6(2)$ & $4.2(3)$ & $4.3(2)$ \\
$R_{\chi}$ & $1.41(2)$ & $1.28(8)$ & $1.33(4)$ \\
$R_{C}$ & $0.173(3)$ & $0.19(1)$ & $0.184(6)$ \\
$R_{4}$ & $8.2(2)$ & $7.7(3)$ & $7.9(2)$ \\
$R_{\xi}^{+}$ & $0.421(1)$ & $0.425(3)$ & $0.423(2)$ \\
$P_{m}$ & $1.201(5)$ & $1.17(2)$ & $1.18(1)$ \\
$P_{c}$ & $0.357(5)$ & $0.357(5)$ \\
$R_{p}$ & $0.354(4)$ & $2.020(5)$ & $2.021(7)$ \\
$z_{\max }$ & $1.275(5)$ & $1.275(6)$ \\
$x_{\max }$ & $2.026(6)$ & $8.3(4)$ & $8.5(2)$ \\
$w_{\max }$ & $1.278(5)$ & $1.53(6)$ & $1.57(4)$ \\
$F_{0}^{\infty}$ & $8.9(1)$ & $0.0266(5)$ & $0.0266(5)$ \\
$r_{8}$ & $1.64(2)$ & $* 0.6(2)$ & $* 0.6(2)$ \\
$r_{10}$ & $-0262(4)$ & $-6(2)$ & $-7(3)$ \\
$f_{1}^{0}$ & $0.23(5)$ & $1.36(5)$ & $1.33(3)$ \\
$f_{2}^{0}$ & $-1.1(3)$ & $0.21(2)$ & $0.20(1)$ \\
$f_{3}^{0}$ & $1.28(1)$ & $-0.105(2)$ & $-0.094(5)$ \\
$c_{f}$ & $0.181(5)$ & $5(3)$ & $8(3)$ \\
\hline \hline & $-0.102(1)$ & &
\end{tabular}

crossover line, $F_{0}^{\infty}$, cf. Eq. (38), which is related to the large- $z$ behavior of $F(z), r_{8}$ and $r_{10}$, cf. Eqs. (36) and (37), which are related to the small- $z$ expansion of $F(z), f_{1}^{0}, f_{2}^{0}$, and $f_{3}^{0}$, cf. Eq. (29), which are related to the expansion at $x=0$ of $f(x)$, and $c_{f}$, cf. Eq. (31), which is related to the behavior at the coexistence curve. Note that $f_{0}^{\infty}=R_{\chi}^{-1}$ where $f_{0}^{\infty}$ is related to the large- $x$ behavior of $f(x)$, cf. Eq. (30).

From the results of Table $\nabla T 1$ we arrive at the final estimates denoted by IHT-PR in Table $\nabla I I$, obtained by taking the weighted average of the results for $n=1$. The error we quote is the sum of the uncertainty induced by the error on the input parameters and of one half of the difference between the two approximations with $n=1$. In most cases these estimates include the results of the $n=0$ approximation. In Table VIII we compare our results with those obtained in other approaches and in experiments [101. We mention that the field-theoretical estimates of $U_{0}$ have been obtained from the analysis of the fixeddimension expansion in the framework of the minimal renormalization without $\epsilon$-expansion [91,93], and from the standard $\epsilon$-expansion to $O\left(\epsilon^{2}\right)$ [92]. The estimate of $U_{0}$ by CRG was obtained using Eq. (33) and the approximate expression for $f(x)$ reported in Refs. [55, 64]. 
TABLE VIII. Estimates of universal amplitude ratios obtained using different approaches. The numbers marked by an asterisk have been obtained by us using the results reported in the corresponding references.

\begin{tabular}{|c|c|c|c|c|c|c|}
\hline & IHT-PR & $d=3$ exp. & $\epsilon$ - exp. & CRG & $\mathrm{HT}$ & experiments \\
\hline$U_{0}$ & $1.56(4)$ & $\begin{array}{l}1.51(4) \text { 91 } \\
1.544 \text { 93 }\end{array}$ & 1.521(22) 92 & *1.823 55, 64 & & $\begin{array}{l}1.50(5) \text { 17 } \\
1.27(9) \quad 29 \\
1.4(4) \quad 94\end{array}$ \\
\hline$\overline{R_{\alpha}}$ & $4.3(3)$ & $\begin{array}{l}* 4.4(4) \quad 91 \\
* 4.46 \text { 93 } \\
*\end{array}$ & $4.56(9)$ 92 & $* 3.41$ [55, 64] & & \\
\hline$R_{\chi}$ & $1.31(7)$ & & 1.33 95 & 1.11 [55,64 & & \\
\hline$\overline{R_{C}}$ & $0.185(10)$ & $\begin{array}{l}0.189(9) \text { 96 } \\
0.194 \text { [93 }\end{array}$ & 0.1797 & & & \\
\hline$R_{4}$ & $7.8(3)$ & & & & & \\
\hline$\overline{R_{\xi}^{+}}$ & $0.424(3)$ & $\begin{array}{l}0.4347(20) \\
0.4319(17) \\
0.100\end{array}$ & 0.42 999 & & $\begin{array}{ll}0.431(5) & 65 \\
0.433(5) & 65\end{array}$ & \\
\hline
\end{tabular}

See, e.g., Ref. 102 for a more complete review of theoretical and experimental estimates of universal amplitude ratios.

In addition, from the approximate parametric representations of the equation of state, we obtain the estimates

$$
\begin{aligned}
& f_{1}^{0}=1.34(5), \\
& f_{2}^{0}=0.20(2) \\
& f_{3}^{0}=-0.10(1) \\
& F_{0}^{\infty}=0.0266(5), \\
& r_{10}=-6(3) .
\end{aligned}
$$

The estimate of $r_{10}$ should be compared with the much less precise HT result $r_{10}=-15(10)$ obtained in Sec. IVA. Concerning the quantities involving amplitudes at the crossover line, we report the estimates

$$
\begin{aligned}
& P_{m}=1.18(2), \\
& P_{c}=0.357(5), \\
& R_{p}=2.020(6), \\
& z_{\max }=1.275(5) .
\end{aligned}
$$

In order to determine the behavior of the longitudinal magnetic susceptibility $\chi_{L}=\partial M / \partial H$ as a function of $t$ and $H$, one may consider the scaling function

$$
\begin{aligned}
& D(w) \equiv B_{c}^{-1} H^{1-1 / \delta} \chi_{L}=\frac{f(x)^{1-1 / \delta}}{\delta f(x)-\frac{1}{\beta} x f^{\prime}(x)}, \\
& w \equiv\left(B / B_{c}\right)^{1 / \beta} t H^{-1 /(\beta \delta)}=x f(x)^{-1 /(\beta \delta)} .
\end{aligned}
$$




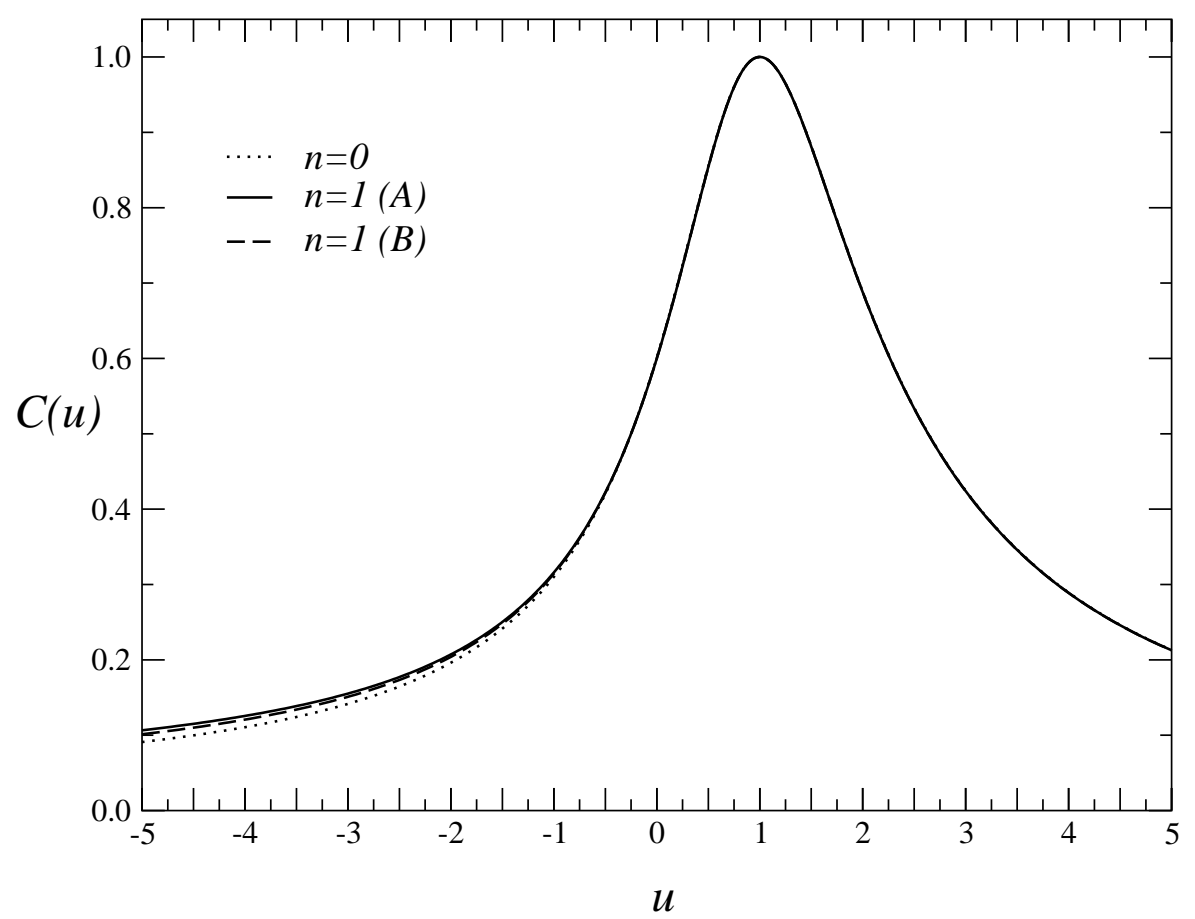

FIG. 3. The scaling function $C(u)$, cf. Eqs. (72) and (71).

The function $D(w)$ has a maximum for $w_{\max }=1.55(6)$. In order to simplify possible comparisons, it is convenient to consider the rescaled function

$$
\begin{aligned}
& C(u)=\frac{D(w)}{D\left(w_{\max }\right)}, \\
& u=\frac{w}{w_{\max }},
\end{aligned}
$$

which is such that the maximum corresponds to $u=1$ and satisfies $C(1)=1$. In Fig. 3 we plot the scaling function $C(u)$ versus $u$, as obtained from the $n=0,1$ approximate parametric representations.

\section{E. Comparison with the experiments}

In spite of the large number of experiments, at present there is no accurate quantitative study of the equation of state in the critical regime. We shall discuss here three different representations that are widely used in the experimental work and we shall give explicit formulae for them.

A first possibility [103 consists in studying the behavior of $h / m \equiv H|t|^{-\gamma} / M$ versus $m^{2}=M^{2}|t|^{-2 \beta}$. Such a function can be easily obtained from our approximations for $f(x)$, since $m^{2}=B^{2}|x|^{-2 \beta}$ and

$$
\frac{h}{m}=k|x|^{-\gamma} f(x)
$$

where the constant $k$ can be written as 


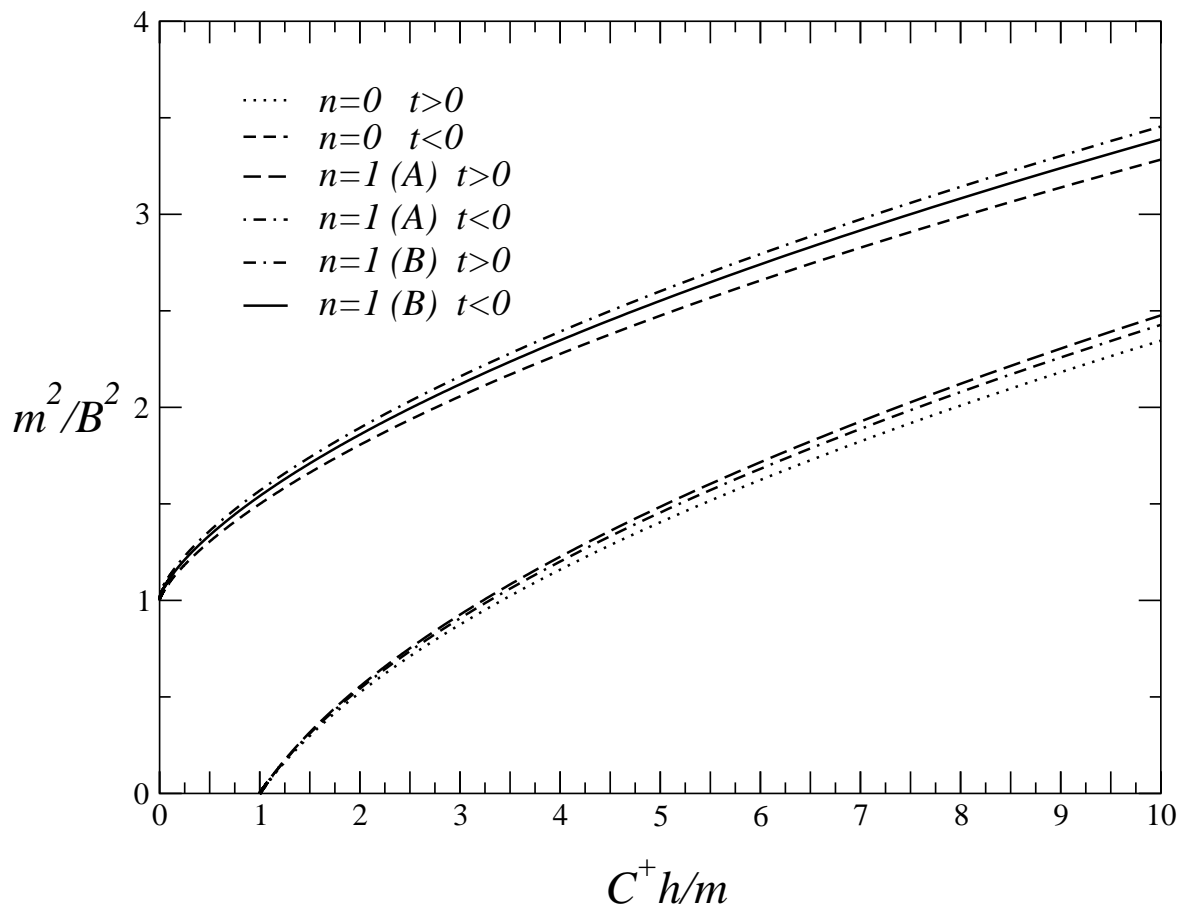

FIG. 4. Plot of $m^{2} / B^{2}$ versus $C^{+} h / m$.

$$
k=\left(B_{c}\right)^{-\delta} B^{\gamma / \beta}=\frac{R_{\chi}}{C^{+}}
$$

The universal ratio $R_{\chi}$ has been computed in the previous section, $R_{\chi}=1.31(7)$, and $B$ and $B_{c}$ are nonuniversal amplitudes defined in Eqs. (28) and (27). A plot of $m^{2} / B^{2}$ versus $C^{+} h / m$ is reported in Fig. 1 . It agrees qualitatively with the analogous experimental ones reported, e.g., in Refs. 17,20,104. Often, for small $h / m$ one approximates the equation of state by writing

$$
\frac{h}{m}=a_{ \pm}+b_{ \pm} m^{2}
$$

where $a_{ \pm}$and $b_{ \pm}$are numerical coefficients depending on the phase. Such an approximation has a very limited range of validity. In the HT phase, we obtain for $m^{2} \rightarrow 0$

$$
\begin{aligned}
\frac{h}{m} & =\frac{1}{C^{+}}\left[1+\frac{R_{4}}{6} \frac{m^{2}}{B^{2}}+\sum_{n=2}^{\infty} \frac{R_{4}^{n} r_{2 n+2}}{(2 n+1) !}\left(\frac{m^{2}}{B^{2}}\right)^{n}\right] \\
& \approx \frac{1}{C^{+}}\left[1+1.30(5) \frac{m^{2}}{B^{2}}+0.94(8)\left(\frac{m^{2}}{B^{2}}\right)^{2}+0.06(2)\left(\frac{m^{2}}{B^{2}}\right)^{3}+\cdots\right],
\end{aligned}
$$

where we have used the estimate of $R_{4}$ reported in Table $\nabla I I I$ and the estimates of $r_{6}$ and $r_{8}$ reported in Table V1. From Eq. $(\overline{76})$ we see that the approximation (75) is valid only for very small $m^{2}$, i.e. at the $1 \%$ level only for $m^{2} \lesssim 0.01 B^{2}$. The quadratic approximationi.e. the approximation with an additional $\left(m^{2}\right)^{2}$ term - has a much wider range of validity because of the smallness of the coefficient of $\mathrm{m}^{6}$. 
In the low-temperature phase, Eq. (75) is theoretically incorrect, since for $m^{2} / B^{2} \rightarrow 1$ we have

$$
\frac{h}{m} \approx \frac{k c_{f}}{4 \beta^{2}}\left(1-\frac{m^{2}}{B^{2}}\right)^{2}
$$

where $c_{f}$ is defined in Eq. (31) and can be estimated roughly from the results reported in Table VTI. Eq. (77) is inconsistent with the approximation (75) near the coexistence curve, due to the presence of Goldstone modes. It would be correct only in Ising systems.

Finally, note that for $m^{2}$ large we have

$$
\frac{h}{m} \approx k\left(\frac{m}{B}\right)^{\delta-1} .
$$

A second form that is widely used to analyze the experimental data is the Arrott-Noakes [105] scaling equation

$$
\left(\frac{H}{M}\right)^{1 / \gamma}=a t+b M^{1 / \beta}
$$

where $a$ and $b$ are numerical constants. This approximation is good in a neighborhood of the critical isotherm $t=0$. Since

$$
\left(\frac{H}{M}\right)^{1 / \gamma} k^{-1 / \gamma}=\left(\frac{M}{B}\right)^{1 / \beta} f(x)^{1 / \gamma}
$$

using Eq. (29) and the numerical values reported in Eq. (66), we obtain

$$
\left(\frac{H}{M}\right)^{1 / \gamma} k^{-1 / \gamma}=\left(\frac{M}{B}\right)^{1 / \beta}+0.96(4) t-0.04(2) t^{2}\left(\frac{M}{B}\right)^{-1 / \beta}-0.02(2) t^{3}\left(\frac{M}{B}\right)^{-2 / \beta} \ldots
$$

Thus, at a $1 \%$ level of precision the Arrott-Noakes formula is valid approximately for $t\left(M B^{-1}\right)^{-1 / \beta} \lesssim 25$ which is quite a large interval.

Finally, Ref. [6] reports an experimental study of the behavior of the critical system at the crossover line, and shows a plot of the curve $C(u)$, cf. Eq. (72), in terms of the unnormalized variable $u_{\exp } \equiv t H^{-1 /(\beta \delta)}$. We can attempt a quantitative comparison with the results reported in their Fig. 4. For this purpose, in Fig. 5 we plot $C(u)$ in terms of their variable for the range of $u_{\exp }$ accessible to the experiment [106]. A direct comparison of this figure with Fig. 4 of Ref. [6] shows a very nice quantitative agreement.

\section{APPENDIX A: MONTE CARLO SIMULATIONS}

In this appendix we present some details of the analyses of the MC data. Details on the simulation can be found in Ref. [37]. 


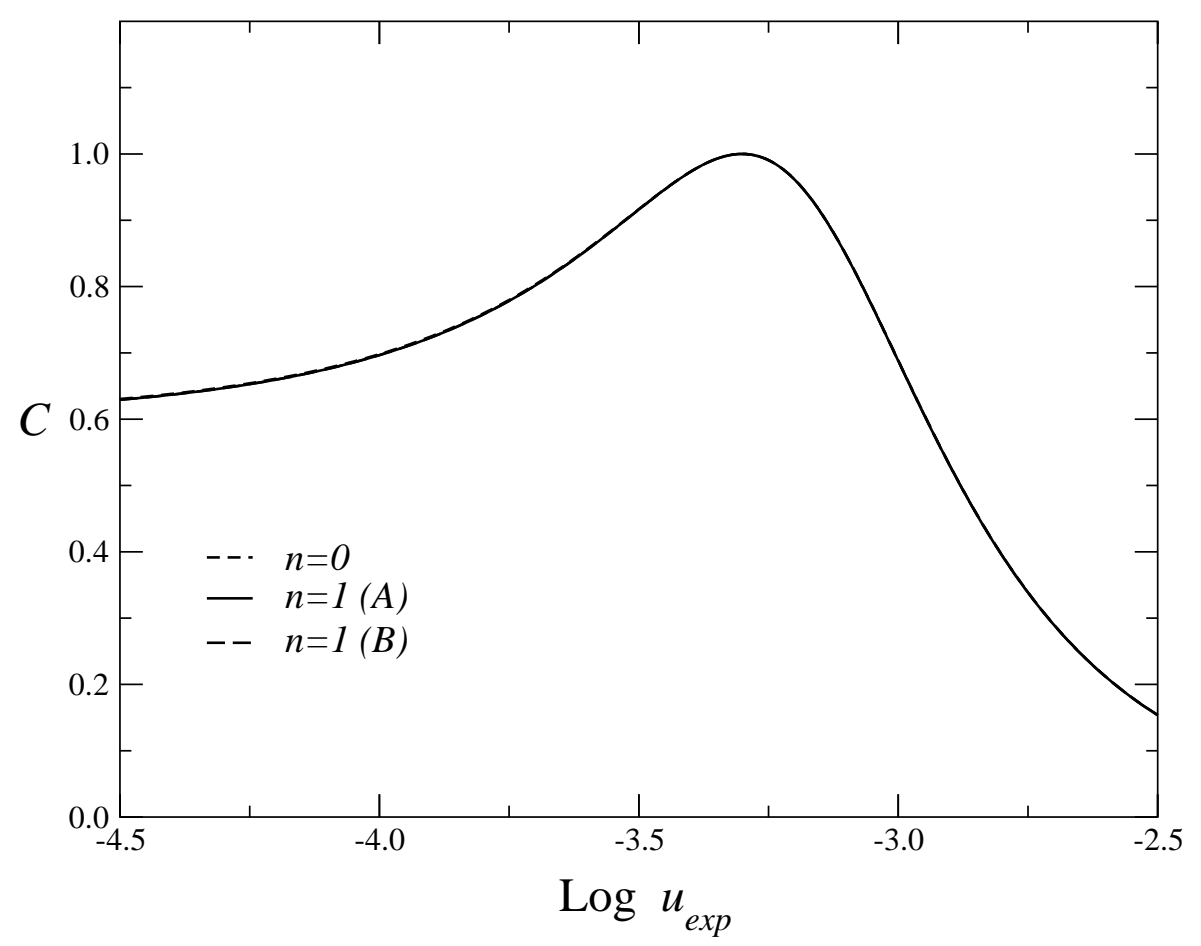

FIG. 5. The scaling function $C(u)$ versus the experimental scaling variable $u_{\exp } \equiv t H^{-1 /(\beta \delta)}$.

\section{Definitions}

In all our work, considerable importance is played by dimensionless ratios (or phenomenological couplings) $R$. In order to have better control on systematic errors we have studied four dimensionless ratios. We first consider the Binder cumulant $U_{4}$ [107] and its generalization $U_{6}$ defined by

$$
U_{2 j}=\frac{\left\langle\left(\vec{m}^{2}\right)^{j}\right\rangle}{\left\langle\vec{m}^{2}\right\rangle^{j}}
$$

where

$$
\vec{m}=\frac{1}{V} \sum_{x} \vec{\phi}_{x}
$$

is the magnetization of the system. The third quantity that we studied is the second-moment correlation length divided by the linear extension of the lattice $\xi_{2 n d} / L$. The second-moment correlation length is defined by

$$
\xi_{2 \mathrm{nd}}=\sqrt{\frac{\chi / F-1}{4 \sin (\pi / L)^{2}}},
$$

where

$$
\chi=\frac{1}{V}\left\langle\left(\sum_{x} \vec{\phi}_{x}\right)^{2}\right\rangle
$$


is the magnetic susceptibility and

$$
F=\frac{1}{V}\left\langle\left|\sum_{x} \exp \left(i \frac{2 \pi x_{1}}{L}\right) \vec{\phi}_{x}\right|^{2}\right\rangle
$$

is the Fourier transform of the two-point correlation function at the lowest nonvanishing momentum. In order to reduce the statistical error, we averaged the results of all three directions of the lattice.

The fourth quantity is the ratio $Z_{a} / Z_{p}$, where $Z_{a}$ is the partition function with antiperiodic boundary conditions in one of the three directions and $Z_{p}$ the corresponding one with periodic boundary conditions in all directions. Anti-periodic boundary conditions mean that the term $\sum_{<x y>} \vec{\phi}_{x} \cdot \vec{\phi}_{y}$ in the Hamiltonian is multiplied by -1 for $x=\left(L_{1}, x_{2}, x_{3}\right)$ and $y=\left(1, x_{2}, x_{3}\right)$. This ratio can be measured with the help of a variant of the cluster algorithm, the boundary-flip algorithm. It was introduced in Ref. [108] for the Ising model and generalized to $O(N)$-invariant nonlinear $\sigma$ models in Ref. [109]. As in Refs. [33, 36], we use a version of the algorithm that only measures $Z_{a} / Z_{p}$ and does not perform the flip to anti-periodic boundary conditions. For a recent discussion of the algorithm, see Ref. [36].

\section{Determination of $R^{*}$}

First, we compute $\beta_{c}$ and the fixed-point value of the dimensionless ratios $R^{*}$ for $\lambda=$ 4.5 - our best approximation to $\lambda^{*}$ - using the standard cumulant crossing method of Binder.

For $\lambda=4.5$ we solve Eq. (2), computing $R(L, \beta)$ by using its Taylor expansion up to the third order:

$$
R(L, \beta)=R\left(L, \beta_{s}\right)+d_{1}\left(L, \beta_{s}\right)\left(\beta-\beta_{s}\right)+\frac{1}{2} d_{2}\left(L, \beta_{s}\right)\left(\beta-\beta_{s}\right)^{2}+\frac{1}{6} d_{3}\left(L, \beta_{s}\right)\left(\beta-\beta_{s}\right)^{3} .
$$

Here $\beta_{s}$ is the value of $\beta$ at which the simulation was performed, and $R, d_{1}, d_{2}$, and $d_{3}$ are determined in the MC simulation.

As an example, results for $Z_{a} / Z_{p}$ are given in Table IX. In the fits, we include all data with $L_{\min } \leq L \leq L_{\max }$. For $L_{\max }=96, \chi^{2} /$ d.o.f. (d.o.f. is the number of degrees of freedom of the fit) is smaller than 1 starting from $L_{\min }=16$. Moreover, the result for $\beta_{c}$ is stable when further data are discarded. To be on the safe side, we take our final estimate from the fit with $L_{\min }=28$ and $L_{\max }=96$.

The systematic error due to corrections to scaling is estimated by comparing the results corresponding to $L_{\min }=28$ and $L_{\max }=96$ with those with $L_{\min }=14$ and $L_{\max }=48$. We suppose that the error of $\beta_{c}$ is proportional to $L^{-1 / \nu-\omega} \approx L^{-2.2}$, where we assume pessimistically leading and not subleading corrections. Hence, we estimate the error on our final result as the difference between the $L_{\min }=28, L_{\max }=96$ result and the $L_{\min }=$ $14, L_{\max }=48$ result multiplied by $2^{-2.2} /\left(1-2^{-2.2}\right)$. The systematic error of $\left(Z_{a} / Z_{p}\right)^{*}$ is estimated in a similar fashion, assuming that the error is proportional to $L^{-\omega}$.

In the same way we analyze our data for the remaining three dimensionless ratios. Our results are reported in Table $\mathrm{IV}$. Note that the four results for $\beta_{c}$ are consistent within error 
TABLE IX. Fits with ansatz (2) of our data for $Z_{a} / Z_{p}$ at $\lambda=4.5$. We included all data with $L_{\min } \leq L \leq L_{\max }$ in the fit.

\begin{tabular}{lcccc}
\hline \hline$L_{\min }$ & $L_{\max }$ & $\chi^{2} /$ d.o.f. & $\beta_{c}$ & $\left(Z_{a} / Z_{p}\right)^{*}$ \\
\hline \hline 12 & 96 & 5.75 & $0.6862428(6)$ & $0.19408(3)$ \\
14 & 96 & 1.90 & $0.6862413(7)$ & $0.19419(3)$ \\
16 & 96 & 1.29 & $0.6862406(7)$ & $0.19424(4)$ \\
18 & 96 & 0.62 & $0.6862400(7)$ & $0.19430(4)$ \\
20 & 96 & 0.68 & $0.6862400(8)$ & $0.19430(5)$ \\
22 & 96 & 0.75 & $0.6862400(8)$ & $0.19430(6)$ \\
24 & 96 & 0.81 & $0.6862397(10)$ & $0.19434(8)$ \\
28 & 96 & 0.60 & $0.6862390(10)$ & $0.19443(10)$ \\
12 & 48 & 5.96 & $0.6862459(10)$ & $0.19399(4)$ \\
14 & 48 & 1.92 & $0.6862432(10)$ & $0.19413(4)$ \\
\hline \hline
\end{tabular}

bars. The statistical error of $\beta_{c}$ obtained from $Z_{a} / Z_{p}$ and $\xi_{2 \text { nd }} / L$ is considerably smaller than that from $U_{4}$ and $U_{6}$. As our final estimate we take $\beta_{c}=0.6862385(20)$, which is consistent with all four results.

\section{Determination of $\lambda^{*}$}

In order to compute $\lambda^{*}$ we solve the equation $\bar{c}(\lambda)=0$, where $\bar{c}(\lambda)$ is defined in Eq. (6). In practice, we replace $\bar{c}(\lambda)$ with its first-order Taylor expansion around $\lambda=4.5$ and thus evaluate $\lambda^{*}$ from

$$
\lambda^{*} \approx 4.5-\bar{c}(4.5)\left(\left.\frac{\mathrm{d} \bar{c}}{\mathrm{~d} \lambda}\right|_{\lambda=4.5}\right)^{-1} .
$$

In order to compute $\bar{c}(4.5)$ we fit our data for $\bar{R}$ with the ansatz (6), where we fix $\omega=0.8$. We have checked that the final result for $\lambda^{*}$ has a very weak dependence on the value of $\omega$ used in the analysis. If we choose $\omega=0.75$, the results vary much less than the quoted error bar, indicating that the error on $\omega$ can be neglected.

As an example, the results for $U_{4}$ at $\left(Z_{a} / Z_{p}\right)_{f}=0.1944$ are given in Table $\mathbf{X}$. We see that there is a slight drift towards larger values of $\bar{c}(4.5)$ as $L_{\min }$ is increased. The final result corresponds to $L_{\min }=16$ and $L_{\max }=96$. Systematic errors due to subleading corrections to scaling are estimated by comparing with the results obtained for $L_{\min }=8$ and $L_{\max }=48$. Assuming the errors on $\bar{c}(4.5)$ to decrease as $L^{-\omega_{2}+\omega} \approx L^{-0.8}$, we arrive at $\bar{c}(4.5)=0.0010(6)[19]$, where the systematic error is quoted in brackets.

In Table X] we give the analogous results for $U_{4}$ at $\left(\xi_{2 \text { nd }} / L\right)_{f}=0.5644$. Here, we see a larger change of $\bar{c}(4.5)$ when $L_{\text {min }}$ is varied. Also, $\chi^{2} /$ d.o.f. is larger for $L_{\min }<14$. Since corrections are larger than above, we take the final estimate from the fit with $L_{\min }=24$ and $L_{\max }=96$. We arrive at the estimate $\bar{c}(4.5)=0.002(2)[5]$. In a similar way we arrive at $\bar{c}(4.5)=0.007(5)[15]$ for $U_{6}$ at $\left(Z_{a} / Z_{p}\right)_{f}=0.1944$ and $\bar{c}(4.5)=0.003(4)[18]$ for $U_{6}$ at $\left(\xi_{2 \mathrm{nd}} / L\right)_{f}=0.5644$. 
TABLE X. Estimates of $\bar{R}^{*}$ and $\bar{c}(4.5)$ from the fit (6) of $U_{4}$ at $\left(Z_{a} / Z_{p}\right)_{f}=0.1944$. Here $\lambda=4.5$.

\begin{tabular}{rlcrr}
\hline \hline$L_{\min }$ & $L_{\max }$ & $\chi^{2} /$ d.o.f. & $\bar{R}^{*}$ & $\bar{c}(4.5)$ \\
\hline \hline 6 & 96 & 7.87 & $1.13931(2)$ & $0.00073(12)$ \\
8 & 96 & 1.71 & $1.13944(2)$ & $-0.00036(17)$ \\
10 & 96 & 1.82 & $1.13942(3)$ & $-0.00022(25)$ \\
12 & 96 & 1.65 & $1.13937(4)$ & $0.00039(36)$ \\
14 & 96 & 1.25 & $1.13930(5)$ & $0.00118(47)$ \\
16 & 96 & 1.33 & $1.13932(5)$ & $0.00097(60)$ \\
18 & 96 & 1.33 & $1.13928(6)$ & $0.00145(73)$ \\
20 & 96 & 1.27 & $1.13923(7)$ & $0.00217(89)$ \\
24 & 96 & 1.54 & $1.13920(10)$ & $0.00273(155)$ \\
8 & 32 & 1.00 & $1.13947(3)$ & $-0.00060(19)$ \\
8 & 48 & 1.26 & $1.13945(2)$ & $-0.00045(18)$ \\
12 & 48 & 1.20 & $1.13939(4)$ & $0.00018(38)$ \\
16 & 48 & 0.75 & $1.13936(6)$ & $0.00056(67)$ \\
\hline \hline
\end{tabular}

TABLE XI. Estimates of $\bar{R}^{*}$ and $\bar{c}(4.5)$ from the fit (6) of $U_{4}$ at $\left(\xi_{2 \text { nd }} / L\right)_{f}=0.5644$. Here $\lambda=4.5$.

\begin{tabular}{rlrrr}
\hline \hline$L_{\min }$ & $L_{\max }$ & $\chi^{2} /$ d.o.f. & \multicolumn{1}{c}{$\bar{R}^{*}$} & $\bar{c}(4.5)$ \\
\hline \hline 8 & 96 & 9.98 & $1.13984(2)$ & $-0.00493(19)$ \\
10 & 96 & 5.05 & $1.13966(3)$ & $-0.00313(31)$ \\
12 & 96 & 2.32 & $1.13948(4)$ & $-0.00120(39)$ \\
14 & 96 & 1.30 & $1.13937(5)$ & $0.00010(51)$ \\
16 & 96 & 1.40 & $1.13936(6)$ & $0.00022(65)$ \\
18 & 96 & 1.21 & $1.13929(7)$ & $0.00112(81)$ \\
20 & 96 & 1.24 & $1.13925(8)$ & $0.00167(99)$ \\
24 & 96 & 1.50 & $1.13923(11)$ & $0.00209(173)$ \\
8 & 48 & 10.41 & $1.13987(3)$ & $-0.00514(19)$ \\
12 & 48 & 1.99 & $1.13952(4)$ & $-0.00153(42)$ \\
\hline \hline
\end{tabular}

Next, we compute $\mathrm{d} \bar{c} / \mathrm{d} \lambda$ at $\lambda=4.5$. To estimate the derivative of $\bar{c}(\lambda)$, we consider the finite differences

$$
\left.\frac{\mathrm{d} \bar{c}}{\mathrm{~d} \lambda}\right|_{\lambda=4.5} \approx[\bar{R}(L, 5.0)-\bar{R}(L, 4.0)] L^{\omega}
$$

The results for our four choices of $\bar{R}$ are given in Table XII. We see that the results, as functions of $L$, are constant within error bars. This nicely confirms the exponent $\omega=0.8$.

The final result for the derivative is obtained by averaging the results for $L \geq 12$, see Table XIII. In order to estimate the discretization error, we additionally compute the derivative, using $\bar{R}(L, \lambda)$ for the pair $\lambda=5.0$ and $\lambda=4.5$. The difference with the abovereported result is small and in practice negligible, approximately $12 \%$ for $U_{4}$ at $\left(Z_{a} / Z_{p}\right)_{f}=$ 
TABLE XII. Estimates of $[\bar{R}(L, 5.0)-\bar{R}(L, 4.0)] L^{\omega}$ with $\omega=0.8$.

\begin{tabular}{rllcl}
\hline \hline$L$ & $U_{4}$ at $\left(Z_{a} / Z_{p}\right)_{f}$ & $U_{4}$ at $\left(\xi_{2 \text { nd }} / L\right)_{f}$ & $U_{6}$ at $\left(Z_{a} / Z_{p}\right)_{f}$ & $U_{6}$ at $\left(\xi_{2 \text { nd }} / L\right)_{f}$ \\
\hline \hline 6 & $-0.00957(17)$ & $-0.01128(18)$ & $-0.03141(53)$ & $-0.03674(54)$ \\
8 & $-0.00951(21)$ & $-0.01136(22)$ & $-0.03111(67)$ & $-0.03697(70)$ \\
10 & $-0.00973(25)$ & $-0.01174(27)$ & $-0.03164(80)$ & $-0.03799(85)$ \\
12 & $-0.00972(28)$ & $-0.01160(30)$ & $-0.03150(88)$ & $-0.03747(93)$ \\
14 & $-0.00983(33)$ & $-0.01188(36)$ & $-0.03201(105)$ & $-0.03855(113)$ \\
16 & $-0.00972(37)$ & $-0.01181(40)$ & $-0.03183(115)$ & $-0.03848(123)$ \\
18 & $-0.00972(47)$ & $-0.01195(53)$ & $-0.03177(148)$ & $-0.03884(162)$ \\
20 & $-0.01022(57)$ & $-0.01174(63)$ & $-0.03392(179)$ & $-0.03880(194)$ \\
22 & $-0.00947(68)$ & $-0.01176(74)$ & $-0.03074(213)$ & $-0.03795(231)$ \\
24 & $-0.00962(79)$ & $-0.01209(86)$ & $-0.03128(249)$ & $-0.03915(272)$ \\
28 & $-0.00962(124)$ & $-0.01159(135)$ & $-0.03208(388)$ & $-0.03832(423)$ \\
\hline \hline
\end{tabular}

TABLE XIII. Results for $[\bar{R}(L, 5.0)-\bar{R}(L, \lambda)] /(5.0-\lambda) L^{\omega}$ with $\omega=0.8$ for $L \geq 12$. In the first row $\lambda=4.0$, in the second one $\lambda=4.5$.

\begin{tabular}{lccc}
\hline \hline$U_{4}$ at $\left(Z_{a} / Z_{p}\right)_{f}$ & $U_{4}$ at $\left(\xi_{2 \text { nd }} / L\right)_{f}$ & $U_{6}$ at $\left(Z_{a} / Z_{p}\right)_{f}$ & $U_{6}$ at $\left(\xi_{2 \text { nd }} / L\right)_{f}$ \\
\hline \hline$-0.00976(16)$ & $-0.01177(17)$ & $-0.03184(49)$ & $-0.03823(53)$ \\
$-0.00872(27)$ & $-0.01035(29)$ & $-0.02838(86)$ & $-0.03356(92)$ \\
\hline \hline
\end{tabular}

0.1944 and $U_{4}$ at $\left(\xi_{2 \text { nd }} / L\right)_{f}=0.5644$, and approximately $14 \%$ for $U_{6}$ at $\left(Z_{a} / Z_{p}\right)_{f}=0.1944$ and $U_{6}$ at $\left(\xi_{2 \text { nd }} / L\right)_{f}=0.5644$.

Inserting our numerical results for $\bar{c}(4.5)$ and $\mathrm{d} \bar{c} / \mathrm{d} \lambda$ into Eq. (A7) we get $\lambda^{*}=4.6(4)$, $4.7(8), 4.7(8)$, and $4.6(8)$ from $U_{4}$ at $\left(Z_{a} / Z_{p}\right)_{f}=0.1944, U_{4}$ at $\left(\xi_{2 \mathrm{nd}} / L\right)_{f}=0.5644, U_{6}$ at $\left(Z_{a} / Z_{p}\right)_{f}=0.1944$ and $U_{6}$ at $\left(\xi_{2 \text { nd }} / L\right)_{f}=0.5644$, respectively. The errors take into account the uncertainty of $\bar{c}(4.5),\left.\frac{\mathrm{d} \bar{c}}{\mathrm{~d} \lambda}\right|_{\lambda=4.5}$, and $\omega$.

As our final result we quote $\lambda^{*}=4.6(4)$ from $U_{4}$ at $\left(Z_{a} / Z_{p}\right)_{f}=0.1944$.

\section{Critical exponents}

We compute the critical exponents $\nu$ and $\eta$ using standard FSS methods.

\section{a. The exponent $\nu$}

The exponent $\nu$ is determined by fitting the data with Eq. (8). We study the derivative of all four quantities $U_{4}, U_{6}, \xi_{\text {2nd }} / L$, and $Z_{a} / Z_{p}$, and fix $\beta_{f}$ by using either $\left(\xi_{\text {2nd }} / L\right)_{f}=0.5644$ or $\left(Z_{a} / Z_{p}\right)_{f}=0.1944$.

As typical examples, we give fit results for $\left.\frac{\partial U_{4}}{\partial \beta}\right|_{\beta_{f}},\left.\frac{\partial\left(Z_{a} / Z_{p}\right)}{\partial \beta}\right|_{\beta_{f}}$ and $\left.\frac{\partial\left(\xi_{\text {2nd }} / L\right)}{\partial \beta}\right|_{\beta_{f}}$ in Tables $\mathrm{XIV}, \mathrm{XV}$, and $\mathrm{XV}$, respectively. In all these three cases, we have fixed $\beta_{f}$ by $\left(Z_{a} / Z_{p}\right)_{f}$. Fixing $\beta_{f}$ by $\left(\xi_{2 \text { nd }} / L\right)_{f}$ leads to similar results. 
TABLE XIV. Estimates of $\nu$ from the fit of $\left.\frac{\partial U_{4}}{\partial \beta}\right|_{\beta_{f}}$ with ansatz (8). $\beta_{f}$ is fixed by $\left(Z_{a} / Z_{p}\right)_{f}=0.1944$.

\begin{tabular}{rlcc}
\hline \hline$L_{\min }$ & $L_{\max }$ & $\chi^{2} /$ d.o.f. & $\nu$ \\
\hline \hline 6 & 96 & 1.36 & $0.71215(16)$ \\
8 & 96 & 0.72 & $0.71174(20)$ \\
10 & 96 & 0.70 & $0.71179(26)$ \\
12 & 96 & 0.71 & $0.71160(34)$ \\
16 & 96 & 0.79 & $0.71137(49)$ \\
20 & 96 & 0.86 & $0.71167(66)$ \\
\hline \hline
\end{tabular}

TABLE XV. Estimates of $\nu$ from the fit of $\left.\frac{\partial\left(Z_{a} / Z_{p}\right)}{\partial \beta}\right|_{\beta_{f}}$ with the ansatz (8). $\beta_{f}$ is fixed by $\left(Z_{a} / Z_{p}\right)_{f}=0.1944$.

\begin{tabular}{rlrl}
\hline \hline$L_{\min }$ & $L_{\max }$ & $\chi^{2} /$ d.o.f. & \multicolumn{1}{c}{$\nu$} \\
\hline \hline 6 & 96 & 69.37 & $0.70660(6)$ \\
8 & 96 & 11.95 & $0.70837(8)$ \\
10 & 96 & 3.28 & $0.70918(11)$ \\
12 & 96 & 1.91 & $0.70969(15)$ \\
16 & 96 & 1.40 & $0.71009(22)$ \\
20 & 96 & 1.21 & $0.71054(30)$ \\
24 & 96 & 1.46 & $0.71044(46)$ \\
28 & 96 & 1.59 & $0.71071(58)$ \\
\hline \hline
\end{tabular}

In the case of $\left.\frac{\partial U_{4}}{\partial \beta}\right|_{\beta_{f}}$ we see that $\chi^{2} /$ d.o.f. is close to one, even if all lattice sizes $L \geq 6$ are included in the fit. Also, the result for $\nu$ stays rather stable when $L_{\min }$ is increased.

On the other hand, for $\left.\frac{\partial\left(Z_{a} / Z_{p}\right)}{\partial \beta}\right|_{\beta_{f}}$ and $\left.\frac{\partial\left(\xi_{\text {2nd }} / L\right)}{\partial \beta}\right|_{\beta_{f}}, \chi^{2} /$ d.o.f. comes close to one only starting from $L_{\min } \geq 16$. Also, the results for $\nu$ strongly change, when $L_{\min }$ is increased. It is interesting to notice that the estimate of $\nu$ is decreasing for $\left.\frac{\partial\left(\xi_{2 n d} / L\right)}{\partial \beta}\right|_{\beta_{f}}$ while it is increasing for $\left.\frac{\partial\left(Z_{a} / Z_{p}\right)}{\partial \beta}\right|_{\beta_{f}}$ when $L_{\min }$ is increased. Assuming that this is already the asymptotic behavior, we can find lower and upper bounds for $\nu$.

Taking into account the fit results for $L_{\min } \geq 22$ we arrive at the final estimate $\nu=$ 0.7113(10). Here, the error bar includes both the statistical and the systematic error.

Finally, we try to determine the effect of leading corrections to scaling on our estimate of $\nu$. For this purpose we fit our data up to $L_{\max }=28$ at $\lambda=4.0, \lambda=4.5$, and $\lambda=5.0$ with the ansatz (8). In Table XVI] we give our results for the derivative of the Binder cumulant with respect to $\beta$ at $\left(Z_{a} / Z_{p}\right)_{f}=0.1944$. In particular, for small $L_{\text {min }}$, we see a clear dependence of the result for $\nu$ on $\lambda$. For instance, for $L_{\min }=8$ the difference between the result for $\lambda=4.0$ and $\lambda=5.0$ is $0.00176(46)$.

In Table XVIII we give the corresponding analysis for $Z_{a} / Z_{p}$ at $\left(Z_{a} / Z_{p}\right)_{f}=0.1944$. In 
TABLE XVI. Estimates of $\nu$ from the fit of $\left.\frac{\partial\left(\xi_{2 \text { nd }} / L\right)}{\partial \beta}\right|_{\beta_{f}}$ with ansatz (8). $\beta_{f}$ is fixed by $\left(Z_{a} / Z_{p}\right)_{f}=0.1944$.

\begin{tabular}{rrrr}
\hline \hline$L_{\min }$ & $L_{\max }$ & $\chi^{2} /$ d.o.f. & \multicolumn{1}{c}{$\nu$} \\
\hline \hline 6 & 96 & 68.10 & $0.71826(8)$ \\
8 & 96 & 18.76 & $0.71613(11)$ \\
10 & 96 & 7.72 & $0.71489(14)$ \\
12 & 96 & 4.35 & $0.71394(18)$ \\
16 & 96 & 1.32 & $0.71261(27)$ \\
20 & 96 & 1.43 & $0.71246(37)$ \\
24 & 96 & 1.27 & $0.71169(56)$ \\
28 & 96 & 1.39 & $0.71196(70)$ \\
\hline \hline
\end{tabular}

TABLE XVII. Estimates of $\nu$ computed from the derivative of the Binder cumulant at $\beta_{f}$, where $\beta_{f}$ is fixed by $\left(Z_{a} / Z_{p}\right)_{f}=0.1944$.

\begin{tabular}{|c|c|c|c|}
\hline$L_{\min }$ & $L_{\max }$ & $\chi^{2} /$ d.o.f. & $\nu$ \\
\hline \multicolumn{4}{|c|}{$\lambda=4.0$} \\
\hline 6 & 28 & 0.91 & $0.71301(24)$ \\
\hline 8 & 28 & 0.75 & $0.71269(33)$ \\
\hline 10 & 28 & 0.83 & $0.71296(44)$ \\
\hline 12 & 28 & 1.07 & $0.71275(64)$ \\
\hline \multicolumn{4}{|c|}{$\lambda=4.5$} \\
\hline 6 & 28 & 1.64 & $0.71226(17)$ \\
\hline 8 & 28 & 0.74 & $0.71181(23)$ \\
\hline 10 & 28 & 0.67 & $0.71193(32)$ \\
\hline 12 & 28 & 0.74 & $0.71170(46)$ \\
\hline \multicolumn{4}{|c|}{$\lambda=5.0$} \\
\hline 6 & 28 & 1.27 & $0.71153(23)$ \\
\hline 8 & 28 & 0.76 & $0.71093(32)$ \\
\hline 10 & 28 & 0.44 & $0.71034(43)$ \\
\hline 12 & 28 & 0.51 & $0.71014(60)$ \\
\hline
\end{tabular}

this case we see a much smaller dependence of the results for $\nu$ on $\lambda$. For $L_{\min }=8$ the difference between the results for $\lambda=4.0$ and $\lambda=5.0$ is $-0.00065(18)$. The behavior in the case of $\xi_{2 n d} / L$ (which is not shown here) is much the same: the dependence of the fit result for $\nu$ on $\lambda$ is much smaller than for the Binder cumulant.

Taking into account the range the lattice sizes that are used to obtain the final result for $\nu$, we arrive at a possible uncertainty of 0.0001 for $\nu$ from the derivative of $Z_{a} / Z_{p}$ and of $\xi_{2 \text { nd }} / L$ due to the uncertainty in $\lambda^{*}$. The systematic error is clearly dominated by subleading corrections. Our final MC estimate of $\nu$ is

$$
\nu=0.7113(11)
$$


TABLE XVIII. Estimates of $\nu$ computed from the derivative of $Z_{a} / Z_{p}$ at $\beta_{f}$, where $\beta_{f}$ is fixed by $\left(Z_{a} / Z_{p}\right)_{f}=0.1944$.

\begin{tabular}{|c|c|c|c|}
\hline$L_{\min }$ & $L_{\max }$ & $\chi^{2} /$ d.o.f. & $\nu$ \\
\hline \multicolumn{4}{|c|}{$\lambda=4.0$} \\
\hline 8 & 28 & 8.97 & $0.70766(13)$ \\
\hline 10 & 28 & 1.63 & $0.70871(18)$ \\
\hline 12 & 28 & 1.58 & $0.70902(27)$ \\
\hline \multicolumn{4}{|c|}{$\lambda=4.5$} \\
\hline 8 & 28 & 14.38 & $0.70804(9)$ \\
\hline 10 & 28 & 2.84 & $0.70890(13)$ \\
\hline 12 & 28 & 1.57 & $0.70941(19)$ \\
\hline \multicolumn{4}{|c|}{$\lambda=5.0$} \\
\hline 8 & 28 & 8.52 & $0.70831(13)$ \\
\hline 10 & 28 & 2.89 & $0.70916(18)$ \\
\hline 12 & 28 & 2.40 & $0.70965(25)$ \\
\hline
\end{tabular}

TABLE XIX. Estimates of $\eta$ from fits of the magnetic susceptibility at $\lambda=4.5$ with Eq. (10). $\beta_{f}$ is fixed by $\left(Z_{a} / Z_{p}\right)_{f}=0.1944$.

\begin{tabular}{lcrc}
\hline \hline$L_{\min }$ & $L_{\max }$ & $\chi^{2} /$ d.o.f. & $\eta$ \\
\hline \hline 12 & 96 & 32.55 & $0.03557(5)$ \\
16 & 96 & 6.36 & $0.03641(7)$ \\
20 & 96 & 1.73 & $0.03682(9)$ \\
24 & 96 & 1.00 & $0.03710(13)$ \\
28 & 96 & 0.81 & $0.03725(16)$ \\
32 & 96 & 0.81 & $0.03740(24)$ \\
\hline \hline
\end{tabular}

\section{b. The exponent $\eta$}

We determine the exponent $\eta$ by using Eq. (10) and also a fit ansatz that includes a constant background term, Eq. (11). We fix $\beta_{f}$ by setting either $\left(\xi_{2 \text { nd }} / L\right)_{f}=0.5644$ or $\left(Z_{a} / Z_{p}\right)_{f}=0.1944$.

Fits for $\lambda=4.5$ with the ansatz (10) are given in Table XIX $\left(\beta_{f}\right.$ fixed by $\left(Z_{a} / Z_{p}\right)_{f}=$ $0.1944)$ and $\mathrm{XX}\left(\beta_{f}\right.$ fixed by $\left.\left(\xi_{2 n d} / L\right)_{f}=0.5644\right)$. In both cases, $\chi^{2} /$ d.o.f. becomes close to one starting from $L_{\text {min }}=24$. Moreover, in both cases the fit results for $\eta$ are strongly increasing as $L_{\min }$ is increased. For $L_{\min }=32$ we have a consistent result of $\eta=0.0374(2)$.

Next, we checked the dependence of the result for $\eta$ on $\lambda$. In Table XXI we give results for $\lambda=4.0,4.5$, and 5.0 for $L_{\min }=16$ and $L_{\max }=28$. We see a rather strong dependence on $\lambda$. The difference between the results for $\lambda=4.0$ and 5.0 is $0.00080(25)$. Taking into account the range of lattice sizes used to obtain our final estimate, we arrive at an error of 0.0002 on $\eta$ due to the error on $\lambda^{*}$.

Finally, we performed fits with the ansatz (11). The results are summarized in Tables 
TABLE XX. Estimates of $\eta$ from fits of the magnetic susceptibility at $\lambda=4.5$ with Eq. (10). $\beta_{f}$ is fixed by $\left(\xi_{2 \mathrm{nd}} / L\right)_{f}=0.5644$.

\begin{tabular}{lcrl}
\hline \hline$L_{\min }$ & $L_{\max }$ & $\chi^{2} /$ d.o.f. & \multicolumn{1}{c}{$\eta$} \\
\hline \hline 12 & 96 & 33.81 & $0.03592(4)$ \\
16 & 96 & 8.24 & $0.03656(5)$ \\
20 & 96 & 2.35 & $0.03695(7)$ \\
24 & 96 & 0.77 & $0.03725(10)$ \\
28 & 96 & 0.59 & $0.03735(12)$ \\
32 & 96 & 0.65 & $0.03742(18)$ \\
\hline \hline
\end{tabular}

TABLE XXI. Estimates of $\eta$ from fits of the magnetic susceptibility at $\lambda=4.0,4.5,5.0$ with Eq. (10). $\beta_{f}$ is fixed by $\left(Z_{a} / Z_{p}\right)_{f}=0.1944$.

\begin{tabular}{lcccc}
\hline \hline$\lambda$ & $L_{\min }$ & $L_{\max }$ & $\chi^{2} /$ d.o.f. & $\eta$ \\
\hline \hline 4.0 & 16 & 28 & 4.75 & $0.03610(18)$ \\
4.5 & 16 & 28 & 2.96 & $0.03562(13)$ \\
5.0 & 16 & 28 & 8.53 & $0.03530(18)$ \\
\hline \hline
\end{tabular}

TABLE XXII. Estimates of $\eta$ from fits of the magnetic susceptibility at $\lambda=4.5$ with Eq. (11). $\beta_{f}$ is fixed by $\left(Z_{a} / Z_{p}\right)_{f}=0.1944$.

\begin{tabular}{rllll}
\hline \hline$L_{\min }$ & $L_{\max }$ & $\chi^{2} /$ d.o.f. & \multicolumn{1}{c}{$\eta$} & \multicolumn{1}{c}{$b$} \\
\hline \hline 8 & 96 & 2.18 & $0.03832(8)$ & $-0.657(9)$ \\
10 & 96 & 1.22 & $0.03811(10)$ & $-0.617(17)$ \\
12 & 96 & 0.72 & $0.03790(12)$ & $-0.555(26)$ \\
16 & 96 & 0.66 & $0.03782(17)$ & $-0.528(54)$ \\
\hline \hline
\end{tabular}

XXII and XXIII. We observe that a $\chi^{2} /$ d.o.f. close to one is already reached for $L_{\min }=10$. Moreover, the result for $\eta$ changes little with increasing $L_{\min }$. For $L_{\min }=16$ the results obtained by fixing $\beta_{f}$ by $Z_{a} / Z_{p}$ and $\xi_{2 \text { nd }} / L$ agree. Therefore, we give as our final result $\eta=0.0378(6)$. The error bar is such that it includes the result of the fits with ansatz (10).

\section{APPENDIX B: ANALYSIS OF THE HIGH-TEMPERATURE EXPANSIONS}

In this appendix we report a discussion of our HT analyses. It should allow the reader to understand how we determined our estimates and the reliability of the errors we report, which are to some extent subjective. More details on the methods we use are reported in Ref. [36]. 
TABLE XXIII. Estimates of $\eta$ from fits of the magnetic susceptibility at $\lambda=4.5$ with Eq. (11). $\beta_{f}$ is fixed by $\left(\xi_{2 \text { nd }} / L\right)_{f}=0.5644$.

\begin{tabular}{rlccc}
\hline \hline$L_{\min }$ & $L_{\max }$ & $\chi^{2} /$ d.o.f. & $\eta$ & $b$ \\
\hline \hline 8 & 96 & 0.80 & $0.03756(6)$ & $-0.379(6)$ \\
10 & 96 & 0.68 & $0.03765(7)$ & $-0.396(11)$ \\
12 & 96 & 0.64 & $0.03772(9)$ & $-0.416(20)$ \\
16 & 96 & 0.53 & $0.03780(13)$ & $-0.454(39)$ \\
\hline \hline
\end{tabular}

\section{Definitions and HT series}

We computed the HT expansion of several quantities for the $\phi^{4}$ lattice Hamiltonian (1) for generic values of $\lambda$ by using the linked-cluster expansion technique. A general introduction to this technique can be found in Refs. 110 112. We calculated the 20th-order HT expansion of the magnetic susceptibility and of the second moment of the two-point function,

$$
\chi=\sum_{x}\left\langle\phi_{\alpha}(0) \phi_{\alpha}(x)\right\rangle, \quad m_{2}=\sum_{x} x^{2}\left\langle\phi_{\alpha}(0) \phi_{\alpha}(x)\right\rangle,
$$

and therefore, the second-moment correlation length $\xi^{2}=m_{2} /(6 \chi)$. Moreover, we computed the HT expansion of the zero-momentum connected $2 j$-point Green's functions $\chi_{2 j}$

$$
\chi_{2 j}=\sum_{x_{2}, \ldots, x_{2 j}}\left\langle\phi_{\alpha_{1}}(0) \phi_{\alpha_{1}}\left(x_{2}\right) \ldots \phi_{\alpha_{j}}\left(x_{2 j-1}\right) \phi_{\alpha_{j}}\left(x_{2 j}\right)\right\rangle_{c}
$$

$\left(\chi=\chi_{2}\right)$. More precisely, we computed $\chi_{4}$ to 18 th order, $\chi_{6}, \chi_{8}$, and $\chi_{10}$ to 15 th order. In Table XXIV] we report the series for the $\phi^{4}$ Hamiltonian with $\lambda=4.5$. We chose this value because it is very close to the best estimate of $\lambda^{*}$, and because for this value of $\lambda$ we have a precise MC estimate of $\beta_{c}, \beta_{c}=0.6862385(20)$.

The HT series of the zero-momentum four-point coupling $g_{4}$ and of the coefficients $r_{2 j}$ that parametrize the small-magnetization expansion of the equation of state can be computed using their definitions in terms of $\chi_{2 j}$ and $\xi^{2}$, i.e.,

$$
g_{4}=-\frac{3 N}{N+2} \frac{\chi_{4}}{\chi^{2} \xi^{3}},
$$

and

$$
\begin{aligned}
r_{6}= & 10-\frac{5(N+2)}{3(N+4)} \frac{\chi_{6} \chi_{2}}{\chi_{4}^{2}}, \\
r_{8}= & 280-\frac{280(N+2)}{3(N+4)} \frac{\chi_{6}}{\chi_{4}^{2}}+\frac{35(N+2)^{2}}{9(N+4)(N+6)} \frac{\chi_{8} \chi_{2}^{2}}{\chi_{4}^{3}}, \\
r_{10}= & 15400-\frac{7700(N+2)}{(N+4)} \frac{\chi_{6} \chi_{2}}{\chi_{4}^{2}}+\frac{350(N+2)^{2}}{(N+4)^{2}} \frac{\chi_{6}^{2} \chi_{2}^{2}}{\chi_{4}^{4}} \\
& +\frac{1400(N+2)^{2}}{3(N+4)(N+6)} \frac{\chi_{8} \chi_{2}^{2}}{\chi_{4}^{3}}-\frac{35(N+2)^{3}}{3(N+4)(N+6)(N+8)} \frac{\chi_{10} \chi_{2}^{3}}{\chi_{4}^{4}} .
\end{aligned}
$$

The formulae relevant for the Heisenberg universality class are obtained setting $N=3$. 
TABLE XXIV. Coefficients of the HT expansion of $m_{2}, \chi, \chi_{4}, \chi_{6}, \chi_{8}$, and $\chi_{10}$, for the $\phi^{4}$ Hamiltonian with $\lambda=4.5$.

\begin{tabular}{|c|c|c|c|}
\hline$i$ & $m_{2}$ & $\chi_{2}$ & $\chi_{4}$ \\
\hline 0 & 0 & 0.95784805390722532625540 & -0.10220686631889066464185 \\
\hline 1 & 0.61164859624923922306340 & 1.83494578874771766919200 & -0.78318918399604286311017 \\
\hline 2 & 2.34346567036967995931352 & 3.02570526555699314952643 & -3.40993303251195614433724 \\
\hline 3 & 6.12631563804734885224064 & 4.91084272357011167693574 & -11.4547261149387426111654 \\
\hline 4 & 13.7411678013148223984708 & 7.72413685583625911405950 & -32.8622857609807070588938 \\
\hline 5 & 28.1806907736271607364126 & 12.0696651111374510782698 & -84.8703770522664536068131 \\
\hline 6 & 54.6110112231156495979858 & 18.5898743639602203433897 & -203.048391944861326183480 \\
\hline 7 & 101.601106321810555490226 & 28.5129469684875374524852 & -458.487070991901596469076 \\
\hline 8 & 183.444896900967255568497 & 43.3910414400431056256283 & -989.113871576954399902821 \\
\hline 9 & 323.515763329983708761073 & 65.8457097219033943862880 & -2056.80132192600410402482 \\
\hline 10 & 560.008592676223985571196 & 99.4318415601856883686915 & -4148.88336851312159356144 \\
\hline 11 & 954.596109677157386424652 & 149.842255361185424824490 & -8158.09343927729005801740 \\
\hline 12 & 1606.62254117132464855356 & 225.053071205898843291857 & -15696.1032586702224741180 \\
\hline 13 & 2674.82852285795544112124 & 337.491827618915172447234 & -29637.6862646277928041257 \\
\hline 14 & 4412.16935517377929 & 504.872666999718714410906 & -55053.5845813513443015904 \\
\hline 15 & 7219.36840082433737483629 & 754.353044416651842398902 & -100803.117025457078176935 \\
\hline 16 & 11729.5598928466629681760 & 1125.02916338959766441444 & -182227.804691700019121686 \\
\hline 17 & 18938.5632727811981324466 & 1676.21770934179961650320 & -325689.103869085626575337 \\
\hline 18 & 30408.8505644977121915813 & 2493.83291987123696064787 & -576156.574987690512078391 \\
\hline 19 & 48583.1353446096892196268 & 3707.29637073719901187394 & \\
\hline 20 & 77271.9486733817666941548 & 5504.79157669035824056791 & \\
\hline$i$ & $\chi_{6}$ & $\chi_{8}$ & $\chi_{10}$ \\
\hline 0 & 0.1685619778291961819 & -0.61605090918722894152663 & 3.90323863425838698945752 \\
\hline 1 & 2.564255760481036314377282 & -15.2300029871348773005564 & 141.589060417753517915167 \\
\hline 2 & 19.75833949883362663601629 & -178.372137615950383513749 & 2318.78554766886373709723 \\
\hline 3 & 107.6153442827461300177955 & -1404.03404775262637194221 & 24643.8854034121990204490 \\
\hline 4 & 470.3177686849044939915090 & -8533.18422765821528578124 & 196815.472836825966956507 \\
\hline 5 & 1762.676494500452240334969 & -43117.7286797414375624292 & 1278183.93414715336465143 \\
\hline 6 & 5892.468315770018895075220 & -189462.996356270457135473 & 7085909.43897471702725255 \\
\hline 7 & 18026.10606034742588181350 & -745826.772293316301527782 & 34639336.4985891651938076 \\
\hline 8 & 51364.40898933529517266700 & -2685696.43890603392620130 & 152792638.179196536855095 \\
\hline 9 & 138079.9439575146969655288 & -8982884.52144712077544905 & 618543549.844101982857471 \\
\hline 10 & 353553.4243627006337358070 & -28231531.3374765513159336 & 2328085189.97290506149579 \\
\hline 11 & 868613.6815956677724696610 & -84122578.3791382746491922 & 8229819042.53291083435060 \\
\hline 12 & 2059460.213048375196521201 & -239356596.354404154637789 & 27546203927.2418206714112 \\
\hline 13 & 4734189.661963741454010320 & -654084831.403386579980857 & 87876514492.4881461446469 \\
\hline 14 & 10591072.53372872753915110 & -1724768015.94141791734955 & 268647512699.595417711632 \\
\hline 15 & 23130642.47447337362164738 & -4405965912.50799258526090 & 790611696518.867100914318 \\
\hline
\end{tabular}




\section{Critical exponents}

In order to estimate $\gamma$ and $\nu$, we analyzed the 20th-order HT expansion of the magnetic susceptibility and the 19 th-order HT expansion of $\xi^{2} / \beta$. We analyzed the HT series by means of integral approximants [113] (IA's) of first, second, and third order (IA1's, IA2's and IA3's respectively). Since the most precise results are obtained by using the MC estimates of $\beta_{c}$ to bias the approximants, we shall only report the results of the biased analyses. We used the values of $\beta_{c}$ obtained in Sec. A2, i.e.

$$
\begin{aligned}
& \beta_{c}(\lambda=4.0)=0.6843895(35), \\
& \beta_{c}(\lambda=4.5)=0.6862385(20), \\
& \beta_{c}(\lambda=5.0)=0.6875638(37) .
\end{aligned}
$$

We considered several sets of biased IA's, and for each of them we obtained estimates of the critical exponents. In the analysis we followed closely Ref. [36. Thus, in the following we shall heavily refer to it for notations and a more detailed description of the analyses.

Given an $n$ th-order series $f(\beta)=\sum_{i=0}^{n} c_{i} \beta^{i}$, its $k$ th-order integral approximant $\left[m_{k} / m_{k-1} / \ldots / m_{0} / l\right]$ IA $k$ is a solution of the inhomogeneous $k$ th-order linear differential equation

$$
P_{k}(\beta) f^{(k)}(\beta)+P_{k-1}(\beta) f^{(k-1)}(\beta)+\ldots+P_{1}(\beta) f^{(1)}(\beta)+P_{0}(\beta) f(\beta)+R(\beta)=0,
$$

where the functions $P_{i}(\beta)$ and $R(\beta)$ are polynomials of order $m_{i}$ and $l$ respectively, which are determined by the known $n$ th-order small- $\beta$ expansion of $f(\beta)$. We considered two types of biased IA $k$ 's:

(i) The first type of biased IA $k$ 's, which will be denoted by bIA $k$ 's, is obtained by setting

$$
P_{k}(\beta)=\left(1-\beta / \beta_{c}\right) p_{k}(\beta)
$$

where $p_{k}(\beta)$ is a polynomial of order $m_{k}-1$.

(ii) Since on bipartite lattices $\beta=-\beta_{c}$ is also a singular point associated to the antiferromagnetic critical behavior [114], we consider IA $k$ 's with

$$
P_{k}(\beta)=\left(1-\beta^{2} / \beta_{c}^{2}\right) p_{k}(\beta)
$$

where $p_{k}(\beta)$ is a polynomial of order $m_{k}-2$. We shall denote them by $\mathrm{b}_{ \pm} \mathrm{IA} k$ 's.

In our analyses we considered diagonal or quasi-diagonal approximants, since they are expected to give the most accurate results. For each set of IA $k$ 's we calculated the average of the values corresponding to all nondefective IA $k$ 's. Approximants are considered defective when they have singularities close to the real $\beta$ axis near the critical point. We also discarded some nondefective IA's - we call them outliers - whose results are far from the average of the other approximants. All details can be found in the App. B of Ref. [36].

In Tables XXV and XXV1 we report the results for $\gamma$ and $\nu$ respectively, obtained by analyzing the series for $\lambda=4.0,4.5$, and 5.0. There, we also quote the "approximant ratio" $r_{a} \equiv(g-f) / t$, where $t$ is the total number of approximants in the given set, $g$ is the number of nondefective approximants, and $f$ is the number of outliers which are discarded using an 
TABLE XXV. Results for $\gamma$ obtained from the analysis of the 20th-order HT series of $\chi$.

\begin{tabular}{llcc}
\hline \hline$\lambda$ & approximants & $r_{a}$ & $\gamma$ \\
\hline \hline 4.0 & bIA1 & $(35-3) / 48$ & $1.39508(6)[32]$ \\
& bIA2 & $(77-7) / 115$ & $1.39503(16)[33]$ \\
\hline 4.5 & bIA1 & $(36-3) / 48$ & $1.39585(4)[18]$ \\
& b IA1 & $(21-1) / 48$ & $1.39583(4)[18]$ \\
& bIA2 & $(93-11) / 115$ & $1.39580(10)[18]$ \\
& b IA2 & $(84-7) / 100$ & $1.39579(18)[18]$ \\
& bIA3 & $(56-6) / 61$ & $1.39582(5)[19]$ \\
\hline 5.0 & bIA1 & $(34-3) / 48$ & $1.39652(6)[32]$ \\
& bIA2 & $(107-13) / 115$ & $1.39648(7)[34]$ \\
\hline \hline
\end{tabular}

TABLE XXVI. Results for $\nu$ obtained from the analysis of the 19 th-order series of $\xi^{2} / \beta$.

\begin{tabular}{llcl}
\hline \hline$\lambda$ & approximants & $r_{a}$ & $\nu$ \\
\hline \hline 4.0 & bIA1 & $(37-6) / 37$ & $0.71061(1)[14]$ \\
& bIA2 & $(65-3) / 70$ & $0.71055(17)[14]$ \\
\hline 4.5 & bIA1 & $(37-4) / 37$ & $0.71110(3)[8]$ \\
& b $_{ \pm}$IA1 & $(31-3) / 36$ & $0.71111(2)[8]$ \\
& bIA2 & $(67-2) / 70$ & $0.71108(6)[7]$ \\
& b IA2 & $(54-3) / 55$ & $0.71114(3)[8]$ \\
& bIA3 & $(26-2) / 34$ & $0.71110(10)[10]$ \\
\hline 5.0 & bIA1 & $(36-4) / 37$ & $0.71151(5)[15]$ \\
& bIA2 & $(67-5) / 70$ & $0.71154(6)[14]$ \\
\hline \hline
\end{tabular}

algorithm described in App. B of Ref. [36]; $g-f$ is the number of "good" approximants used in the analysis. Notice that $g \gg f$, and $g-f$ is never too small. For each analysis, beside the corresponding estimate, we report two numbers. The number in parentheses, $e_{1}$, is basically the spread of the approximants for $\beta_{c}$ fixed at its MC estimate. It is the standard deviation of the results obtained from all "good" IA's divided by the square root of $r_{a}$, i.e., $e_{1}=\sigma / \sqrt{r_{a}}$. The number in brackets, $e_{2}$, is related to the uncertainty on the value of $\beta_{c}$ and it is estimated by varying $\beta_{c}$ in the range $\left[\beta_{c}-\Delta \beta_{c}, \beta_{c}+\Delta \beta_{c}\right]$.

\section{APPENDIX C: UNIVERSAL AMPLITUDE RATIOS FROM THE PARAMETRIC REPRESENTATION}

In the following we report the expressions of the universal amplitude ratios in terms of the parametric representation (43) of the critical equation of state.

The singular part of the free energy per unit volume can be written as

$$
\mathcal{F}_{\text {sing }}=h_{0} m_{0} R^{2-\alpha} g(\theta),
$$

where $g(\theta)$ is the solution of the first-order differential equation 


$$
\left(1-\theta^{2}\right) g^{\prime}(\theta)+2(2-\alpha) \theta g(\theta)=Y(\theta) h(\theta)
$$

that is regular at $\theta=1$. The function $Y(\theta)$ has been defined in Eq. (44). The longitudinal magnetic susceptibility can be written as

$$
\chi_{L}^{-1}=\frac{h_{0}}{m_{0}} R^{\gamma} g_{2}(\theta), \quad g_{2}(\theta)=\frac{2 \beta \delta \theta h(\theta)+\left(1-\theta^{2}\right) h^{\prime}(\theta)}{Y(\theta)} .
$$

The function $g_{2}(\theta)$ must vanish at $\theta_{0}$ in order to reproduce the predicted behavior at the coexistence curve $\chi_{L} \sim H^{-1 / 2}$, according to

$$
g_{2}(\theta) \sim \theta_{0}-\theta \quad \text { for } \quad \theta \rightarrow \theta_{0}
$$

From Eq. (C3) we see that $g_{2}(\theta)$ satisfies this condition if $h(\theta) \sim\left(\theta_{0}-\theta\right)^{2}$ for $\theta \rightarrow \theta_{0}$.

From the equation of state one can derive universal amplitude ratios of zero-momentum quantities. We consider

$$
\begin{aligned}
& U_{0} \equiv A^{+} / A^{-}=\left(\theta_{0}^{2}-1\right)^{2-\alpha} \frac{g(0)}{g\left(\theta_{0}\right)} \\
& R_{\chi} \equiv \frac{C^{+} B^{\delta-1}}{B_{c}^{\delta}}=\left(\theta_{0}^{2}-1\right)^{-\gamma}\left[m\left(\theta_{0}\right)\right]^{\delta-1}[m(1)]^{-\delta} h(1), \\
& R_{C} \equiv \frac{\alpha A^{+} C^{+}}{B^{2}}=-\alpha(1-\alpha)(2-\alpha)\left(\theta_{0}^{2}-1\right)^{2 \beta}\left[m\left(\theta_{0}\right)\right]^{-2} g(0), \\
& R_{4} \equiv-\frac{C_{4}^{+} B^{2}}{\left(C^{+}\right)^{3}}=\rho^{2}\left[m\left(\theta_{0}\right)\right]^{2}\left(\theta_{0}^{2}-1\right)^{-2 \beta} .
\end{aligned}
$$

Using Eqs. (45) and (47) one can easily derive the expressions of the various coefficients that characterize the asymptotic behavior of the scaling functions $f(x)$ and $F(z)$, such as $c_{f}, f_{i}^{0}$ for $f(x)$ and $F_{i}^{\infty}, r_{2 j}$ for $F(z)$. Concerning the ratios involving amplitudes along the crossover line, one finds

$$
\begin{aligned}
& P_{m} \equiv \frac{T_{p}^{\beta} B}{B_{c}}=x_{\max }^{\beta} f\left(x_{\max }\right)^{-1 / \delta}, \\
& P_{c} \equiv-\frac{T_{p}^{2 \beta \delta} C^{+}}{C_{4}}=F\left(z_{\max }\right)^{-2}, \\
& R_{p} \equiv \frac{C^{+}}{C_{p}}=F^{\prime}\left(z_{\max }\right) .
\end{aligned}
$$

Here $x_{\max }$ and $z_{\max }$ are the values of the scaling variables $x$ and $z$ computed at $\theta_{\max }$, where $\theta_{\max }$ is the solution of the equation

$$
\beta \delta F[z(\theta)] F^{\prime \prime}[z(\theta)]-\gamma F^{\prime}[z(\theta)]^{2}=0 .
$$




\section{REFERENCES}

* $\quad$ Email address: Massimo.Campostrini@df.unipi.it

$\dagger \quad$ Email address: Martin.Hasenbusch@desy.de

$\ddagger \quad$ Email address: Andrea.Pelissetto@roma1.infn.it

$\S \quad$ Email address: Paolo.Rossi@df.unipi.it

- Email address: Ettore.Vicari@df.unipi.it

[1] The isotropic Heisenberg model is a simplified model and neglects several interactions that are present in real materials. Among them, we should mention the cubic anisotropies due to the lattice structure and the dipolar interactions. Even if, in the renormalization-group language, these effects are relevant perturbations of the Heisenberg fixed point [115 118], the new critical exponents are so close to those of the Heisenberg universality class that the difference is experimentally very difficult to observe, see, e.g., Refs. [119,118, 120,56] and references therein.

[2] M. Shaham, J. Barak, U. El-Hanany, W. W. Warren, Jr., Phys. Rev. B 22, 5400 (1980).

[3] M. A. Kobeissi, Phys. Rev. B 24, 2380 (1981).

[4] M. Seeger, S. N. Kaul, H. Kronmüller, and R. Reisser, Phys. Rev. B 51, 12585 (1995).

[5] R. Reisser, R. K. Kremer, and A. Simon, Phys. Rev. B 52, 3546 (1995).

[6] J. H. Zhao, H. P. Kunkel, X. Z. Zhou, G. Williams, and M. A. Subramanian, Phys. Rev. Lett. 83, 219 (1999).

[7] S. G. Barsov, A. L. Getalov, V. P. Koptev, S. A. Kotov, S. M. Mikirtychyants, G. V. Shcherbatov, A. A. Arsenov, and Ya. M. Mukovskii, Physica B 289-290, 81 (2000).

[8] J. H. Zhao, T. Song, H. P. Kunkel, X. Z. Zhou, R. M. Roshko, and G. Williams, J. Phys.: Condens. Matter 126903 (2000).

[9] C. S. Arnold and D. P. Pappas, Phys. Rev. Lett. 85, 5202 (2000).

[10] J. Mira, J. Rivas, A. Butera, L. B. Steren, J. M. García-Beneytez, and M. Vázquez, J. Appl. Phys. 87, 5911 (2000).

[11] C. Bührer, M. Bechmann, M. Fähnle, U. Grünewald, and K. Maier, J. Magn. Magn. Mater. 212, 211 (2000).

[12] T. Brückel, D. Hupfeld, J. Strempfer, W. Caliebe, K. Mettenberger, A. Stunault, N. Bernhoeft, and G. J. McIntyre, Eur. J. Phys. B 19, 475 (2001).

[13] F. Y . Yang, C. L. Chien, X. W. Li, G. Xiao, and A. Gupta, Phys. Rev. B 63, 092403 (2001).

[14] C. S. Hong, W. S. Kim, and N. H. Hur, Phys. Rev. B 63, 092504 (2001).

[15] For some dopings and some divalent cation A a first-order transition has been observed. Moreover, in systems in which the transition appears to be of second order, mean-field critical exponents have been measured. For instance, for $\mathrm{La}_{1-x} \mathrm{Sr}_{x} \mathrm{MnO}_{3}$, a mean-field value for $\beta$ was observed in Refs. [121-123], while an estimate compatible with the Heisenberg value was found in Refs. [124 [127]. For $x=1 / 3$ there also exists [94 an estimate of the exponent $\alpha, \alpha=-0.14 \pm 0.10$, in agreement with the Heisenberg value.

[16] In order to observe the correct exponents, it is essential to consider corrections to scaling in the analysis of the experimental data [24]. All results reported in Table I], except those of Ref. [21], have been obtained by assuming scaling corrections of the form $\left(1+a|t|^{\Delta_{1}}+b|t|^{\Delta_{2}}\right)$, with $\Delta_{1}=0.11$ and $\Delta_{2}=0.55$. Two observations are in order here. First, the results of the present paper provide a precise estimate of $\Delta_{1}$, 
$\Delta_{1}=0.1336(15)$. Second, the renormalization group predicts also corrections of order $|t|^{2 \Delta_{1}},|t|^{3 \Delta_{1}}$, etc. which are more relevant than the term $|t|^{\Delta_{2}}$ and should therefore be taken into account in the analysis of the data.

[17] S. N. Kaul and M. Sambasiva Rao, J. Phys.: Condens. Matter 6, 7403 (1994).

[18] M. Sambasiva Rao and S. N. Kaul, J. Magn. Magn. Mater. 140-144, 1567 (1995).

[19] M. Sambasiva Rao and S. N. Kaul, J. Magn. Magn. Mater. 147, 149 (1995).

[20] P. D. Babu and S. N. Kaul, J. Phys.: Condens. Matter 9, 7189 (1997).

[21] M. R. Said, Y. A. Hamam, I. Abu-Alyarayesh, S. Mahmood, J. Magn. Magn. Mater. 195, 679 (1999).

[22] A. Perumal, V. Srinivas, V. V. Rao, and R. A. Dunlap, Physica B 292, 164 (2000).

[23] A. Perumal, V. Srinivas, K. S. Kim, S. C. Yu, V. V. Rao, and R. A. Dunlap, J. Magn. Magn. Mater. 233, 280 (2001).

[24] S. N. Kaul, J. Magn. Magn. Mater. 53, 5 (1985).

[25] A. Pelissetto and E. Vicari, Phys. Rev. B 62, 6393 (2000).

[26] S. N. Kaul, Phys. Rev. B 38, 9178 (1988).

[27] O. Källbäck, S. G. Humble, and G. Malmström, Phys. Rev. B 24, 5214 (1981).

[28] B. Stroka, J. Wosnitza, E. Scheer, H. von Löhneysen, W. Park, and K. Fischer, Z. Phys. B 89, 39 (1992).

[29] M. Marinelli, F. Mercuri, S. Foglietta, and D. P. Belanger, Phys. Rev. B 54, 4087 (1996).

[30] H. G. Ballesteros, L. A. Fernández, V. Martín-Mayor, and A. Muñoz Sudupe, Phys. Lett. B 441, 330 (1998).

[31] H. G. Ballesteros, L. A. Fernández, V. Martín-Mayor, A. Muñoz Sudupe, G. Parisi, and J. J. Ruiz-Lorenzo, J. Phys. A 32, 1 (1999).

[32] M. Hasenbusch, K. Pinn, and S. Vinti, Phys. Rev. B 59, 11471 (1999).

[33] M. Hasenbusch, J. Phys. A 32, 4851 (1999).

[34] M. Hasenbusch and T. Török, J. Phys. A 32, 6361 (1999).

[35] M. Hasenbusch, Habilitationsschrift, Humboldt-Universität zu Berlin, 1999; Int. J. Mod. Phys. C 12, 911 (2001).

[36] M. Campostrini, M. Hasenbusch, A. Pelissetto, P. Rossi, and E. Vicari, Phys. Rev. B 63, 214503 (2001).

[37] M. Hasenbusch, J. Phys. A 34, 8221 (2001).

[38] M. Campostrini, A. Pelissetto, P. Rossi, and E. Vicari, Phys. Rev. E 60, 3526 (1999).

[39] M. Campostrini, A. Pelissetto, P. Rossi, and E. Vicari, Phys. Rev. B 61, 5905 (2000).

[40] P. Butera and M. Comi, Phys. Rev. B 56, 8212 (1997).

[41] A. A. Caparica, A. Bunker, and D. P. Landau, Phys. Rev. B 62, 9458 (2000).

[42] H. G. Ballesteros, L. A. Fernández, V. Martín-Mayor, and A. Muñoz Sudupe, Phys. Lett. B 387, 125 (1996).

[43] C. Holm and W. Janke, Phys. Rev. B 48, 936 (1993); Phys. Lett. A 173, 8 (1993); J. Appl. Phys. 73, 5488 (1993).

[44] P. Peczak, A. M. Ferrenberg, and D. P. Landau, Phys, Rev. B. 43, 6087 (1991).

[45] J. Adler, C. Holm, and W. Janke, Physica A 201, 581 (1993).

[46] F. Jasch and H. Kleinert, J. Math. Phys. 42, 52 (2001).

[47] R. Guida and J. Zinn-Justin, J. Phys. A 31, 8103 (1998).

[48] D. B. Murray and B. G. Nickel, "Revised estimates for critical exponents for the 
continuum $n$-vector model in 3 dimensions," unpublished Guelph University report (1991).

[49] J. C. Le Guillou and J. Zinn-Justin, Phys. Rev. Lett. 39, 95 (1977); Phys. Rev. B 21, 3976 (1980).

[50] V. I. Yukalov and S. Gluzman, Phys. Rev. E 58, 1359 (1998).

[51] H. Kleinert, Phys. Lett. A 264, 357 (2000).

[52] K. E. Newman and E. K. Riedel, Phys. Rev. B 30, 6615 (1984).

[53] O. Bohr, B.-J. Schaefer, and J. Wambach, Int. J. Mod. Phys. A 16, 3823 (2001).

[54] G. v. Gersdorff and C. Wetterich, Phys. Rev. B 64, 054513 (2001).

[55] J. Berges, N. Tetradis, and C. Wetterich, Phys. Rev. Lett. 77, 873 (1996).

[56] A. Pelissetto and E. Vicari, "Critical Phenomena and Renormalization-Group Theory", e-print cond-mat/0012164.

[57] Recently, a model with competing superexchange and double-exchange interactions has been studied [128]. A preliminary analysis for the paramagnetic-ferromagnetic transition gives $\nu=0.720(2)$ and $\gamma=1.438(8)$. While $\nu$ is reasonable agreement with the Heisenberg value, $\gamma$ is significantly higher, so that the identification of this transition as a Heisenberg one is in doubt.

[58] G. A. Baker, Jr., B. G. Nickel, M. S. Green, and D. I. Meiron, Phys. Rev. Lett. 36, 1351 (1977); G. A. Baker, Jr., B. G. Nickel, and D. I. Meiron, Phys. Rev. B 17, 1365 (1978).

[59] K. G. Chetyrkin, S. G. Gorishny, S. A. Larin, and F. V. Tkachov, Phys. Lett. B 132, 351 (1983).

[60] H. Kleinert, J. Neu, V. Schulte-Frohlinde, K. G. Chetyrkin, and S. A. Larin, Phys. Lett. B 272, 39 (1991); Erratum B 319, 545 (1993).

[61] S. Hikami and E. Brézin, J. Phys. A 11, 1141 (1978).

[62] S. Hikami, Nucl. Phys. B 215, 555 (1983).

[63] W. Bernreuther and F. J. Wegner, Phys. Rev. Lett. 57, 1383 (1986).

[64] J. Berges, N. Tetradis, and C. Wetterich, "Non-perturbative renormalization flow in quantum field theory and statistical physics," e-print hep-ph/0005122 (2000).

[65] P. Butera and M. Comi, Phys. Rev. B 60, 6749 (1999).

[66] I. M. Mryglod, I. P. Omelyan, and R. Folk, Phys. Rev. Lett. 86, 3156 (2001).

[67] In the so-called critical-point renormalization method (see Ref. [129] and references therein), given two series $D(x)$ and $E(x)$ that are singular at the same point $x_{0}$, $D(x)=\sum_{i} d_{i} x^{i} \sim\left(x_{0}-x\right)^{-\delta}$ and $E(x)=\sum_{i} e_{i} x^{i} \sim\left(x_{0}-x\right)^{-\epsilon}$, one constructs a new series $F(x)=\sum_{i}\left(d_{i} / e_{i}\right) x^{i}$. The function $F(x)$ is singular at $x=1$ and for $x \rightarrow 1$ behaves as $F(x) \sim(1-x)^{-\phi}$, where $\phi=1+\delta-\epsilon$. Therefore, the difference $\delta-\epsilon$ can be obtained by analyzing the expansion of $F(x)$ by means of biased approximants with a singularity at $x_{c}=1$.

[68] E. Brézin and D. J. Wallace, Phys. Rev. B 7, 1967 (1973).

[69] E. Brézin and J. Zinn-Justin, Phys. Rev. B 14, 3110 (1976).

[70] D. J. Wallace and R. P. K. Zia, Phys. Rev. B 12, 5340 (1975).

[71] L. Schäfer and H. Horner, Z. Phys. B 29, 251 (1978).

[72] I. D. Lawrie, J. Phys. A 14, 2489 (1981).

[73] A. Pelissetto and E. Vicari, Nucl. Phys. B 540, 639 (1999).

[74] M. Barmatz, P. C. Hohenberg, and A. Kornblit, Phys. Rev. B 12, 1947 (1975). 
[75] E. Brézin, D. J. Wallace, and K. G. Wilson, Phys. Rev. Lett. 29, 591 (1972); Phys. Rev. B 7, 232 (1973).

[76] R. Guida and J. Zinn-Justin, Nucl. Phys. B 489, 626 (1997).

[77] M. Caselle, M. Hasenbusch, A. Pelissetto, and E. Vicari, J. Phys. A 34, 2923 (2001).

[78] M. Campostrini, A. Pelissetto, P. Rossi, and E. Vicari, Phys. Rev. B 62, 5843 (2000).

[79] J. Zinn-Justin, Quantum Field Theory and Critical Phenomena, third edition (Clarendon Press, Oxford, 1996).

[80] P. Butera and M. Comi, Phys. Rev. B 58, 11552 (1998).

[81] A. Pelissetto and E. Vicari, Nucl. Phys. B 575, 579 (2000).

[82] A. Pelissetto and E. Vicari, Nucl. Phys. B 519, 626 (1998); Nucl. Phys. B (Proc. Suppl.) 73, 775 (1999).

[83] T. Reisz, Phys. Lett. B 360, 77 (1995).

[84] A. I. Sokolov, E. V. Orlov, V. A. Ul'kov, and S. S. Kashtanov, Phys. Rev. E 60, 1344 (1999).

[85] A. Pelissetto and E. Vicari, Nucl. Phys. B 522, 605 (1998).

[86] N. Tetradis and C. Wetterich, Nucl. Phys. B 422, 541 (1994).

[87] P. Schofield, Phys. Rev. Lett. 22, 606 (1969).

[88] P. Schofield, J. D. Lister, and J. T. Ho, Phys. Rev. Lett. 23, 1098 (1969).

[89] B. D. Josephson, J. Phys. C: Solid State Phys. 2, 1113 (1969).

[90] In the HT phase $t>0, M t^{-\beta}=g\left(H t^{-\beta \delta}\right)$ where $g(\tau)$ is analytic in the complex $\tau$ plane except possibly for two branch cuts on the imaginary axis, for $|\operatorname{Im} \tau|>\tau_{0}$ (Yang-Lee theorem). Thus, the convergence radius is at most $|z|<\left|g\left(i \tau_{0}\right)\right| / k$, where $k$ is the normalization of $z$.

[91] S. A. Larin, M. Mönnigmann, M. Strösser, and V. Dohm, Phys. Rev. B 58, 3394 (1998).

[92] C. Bervillier, Phys. Rev. B 34, 8141 (1986).

[93] H. Kleinert and B. Van den Bossche, Phys. Rev. E 63, 056113 (2001).

[94] C. A. Ramos, H. R. Salva, R. D. Sanchez, M. Tovar, F. Rivadulla, J. Mira, J. RIvas, A. M. Lopez-Quintela, L. Hueso, M. Saint-Paul, P. Lejay, and Y. Tokura, J. Magn. Magn. Mater. 226-230, 582 (2001).

[95] R. Abe and M. Masutani, Progr. Theor. Phys. 59, 672 (1978).

[96] M. Stroesser, S.A. Larin, and V. Dohm, Nucl. Phys. B 540, 654 (1999).

[97] A. Aharony and P. C. Hohenberg, Phys. Rev. B 13, 3081 (1976).

[98] C. Bagnuls and C. Bervillier, Phys. Rev. B 32, 7209 (1985).

[99] C. Bervillier, Phys. Rev. B 14, 4964 (1976).

[100] C. Bervillier and C. Godrèche, Phys. Rev. B 21, 5427 (1980).

[101] The amplitudes $B, B_{c}$, and $C^{+}$are determined in several experiments - see, e.g., Ref. [20] - and thus an estimate of the ratio $R_{\chi}$ is possible. However, these amplitudes have large systematic uncertainties, and we can only obtain a rough estimate, $0.6 \lesssim R_{\chi} \lesssim 2$.

[102] V. Privman, P. C. Hohenberg, and A. Aharony, in Phase Transitions and Critical Phenomena, vol. 14, edited by C. Domb and J. L. Lebowitz (Academic Press, New York, 1991).

[103] J. S. Kouvel and D. S. Rodbell, Phys. Rev. Lett. 18, 215 (1967).

[104] S. F. Fischer, S. N. Kaul, and H. Kronmüller, J. Magn. Magn. Mater. 226-230, 540 (2001). 
[105] A. Arrott and J. E. Noakes, Phys. Rev. Lett. 19, 786 (1967).

[106] We have evaluated $\left(u_{\exp }\right)_{\max }$ (the position of the maximum) from Fig. 4 of Ref. [6]: $\left(u_{\exp }\right)_{\max } \approx 0.0005$. The variable $u$ defined in Sec. IVD is related to $u_{\exp }$ by $u=$ $u_{\exp } /\left(u_{\exp }\right)_{\max }$. Note that the $\left(u_{\exp }\right)_{\max }$ can also be derived from the knowledge of the nonuniversal constants $B, B_{c}$, and $C^{+}$, and using the results of Sec. IVD. Indeed,

$$
\left(u_{\exp }\right)_{\max }=\left(\frac{B_{c}}{B}\right)^{1 / \beta} w_{\max }=\left(\frac{C^{+}}{B_{c}}\right)^{1 / \gamma} w_{\max } R_{\chi}^{-1 / \gamma}=\left(\frac{C^{+}}{B}\right)^{1 /(\beta \delta)} w_{\max } R_{\chi}^{-1 /(\beta \delta)} .
$$

Numerically, using the estimates reported in Sec. IVD, we have $w_{\max }=1.55(6)$, $w_{\max } R_{\chi}^{-1 / \gamma}=1.28(7)$, and $w_{\max } R_{\chi}^{-1 /(\beta \delta)}=1.33(7)$.

[107] K. Binder, Z. Phys. B 43, 119 (1981); Phys. Rev. Lett. 47, 693 (1981).

[108] M. Hasenbusch, Physica A 197, 423 (1993).

[109] A. P. Gottlob and M. Hasenbusch, J. Stat. Phys. 77, 919 (1994).

[110] M. Wortis, "Linked cluster expansion", in Phase Transitions and Critical Phenomena, vol. 3, edited by C. Domb and M. S. Green (Academic Press, London, 1974).

[111] M. Lüscher and P. Weisz, Nucl. Phys. B 300, 325 (1988).

[112] M. Campostrini, J. Stat. Phys. 103, 369 (2001).

[113] For a review see, e.g., A. J. Guttmann, in Phase Transitions and Critical Phenomena, Vol. 13, edited by C. Domb and J. Lebowitz (Academic, New York, 1989).

[114] M. E. Fisher, Philos. Mag. 7, 1731 (1962).

[115] M. E. Fisher, Rev. Mod. Phys. 46, 597 (1974).

[116] A. Aharony, in Phase Transitions and Critical Phenomena, edited by C. Domb and J. Lebowitz (Academic Press, New York, 1976), Vol. 6, p. 357.

[117] A. Aharony and M. E. Fisher, Phys. Rev. B 8, 3323 (1973).

[118] J. M. Carmona, A. Pelissetto, and E. Vicari, Phys. Rev. B 61, 15136 (2000).

[119] A. D. Bruce and A. Aharony, Phys. Rev. B 10, 2078 (1974).

[120] S. Srinath, S. N. Kaul, and M.-K. Sostarich, Phys. Rev. B 62, 11649 (2000).

[121] S. E. Lofland, V. Ray, P. H. Kim, S. M. Bhagat, M. A. Manheimer, and S. D. Tyagi, Phys. Rev. B 55, 2749 (1997).

[122] C. V. Mohan, M. Seeger, H. Kronmüller, P. Murugaraj, and J. Maier, J. Magn. Magn. Mater. 83, 348 (1998).

[123] A. Schwartz, M. Scheffler, and S. M. Anlage, Phys. Rev. B 61, R870 (2000).

[124] M. C. Martin, G. Shirane, Y. Endoh, K. Hirota, Y. Moritomo, and Y. Tokura, Phys. Rev. B 53, 14285 (1996).

[125] R. H. Heffner, L. P. Le, M. F. Hundley, J. J. Neumeier, G. M. Luke, K. Kojima, B. Nachumi, Y. J. Uemura, D. E. MacLaughlin, and S.-W. Cheong, Phys. Rev. Lett. 77, 1869 (1996)

[126] S. E. Lofland, S. M. Bhagat, K. Ghosh, R. L. Greene, S. G. Karabashev, D. A. Shulyatev, A. A. Arsenov, and Y. Mukovskii, Phys. Rev. B 56, 13705 (1997).

[127] K. Ghosh, C. J. Lobb, R. L. Greene, S. G. Karabashev, D. A. Shulyatev, A. A. Arsenov, and Y. Mukovskii, Phys. Rev. Lett. 81, 4740 (1998).

[128] S.-H. Tsai and D. P. Landau, J. Magn. Magn. Mater. 226-230, 650 (2001).

[129] D. L. Hunter and G. A. Baker, Jr., Phys. Rev. B 7, 3346 (1973); B 7, 3377 (1973); B 19, 3808 (1979); M. E. Fisher and H. Au-Yang, J. Phys. A 12, 1677 (1979); Erratum A 
13, 1517 (1980); A. J. Guttmann and G. S. Joyce, J. Phys. A 5, L81 (1972); J. J. Rehr, A. J. Guttmann, and G. S Joyce, J. Phys. A 13, 1587 (1980). 